\title{
Induction of cardiomyocyte proliferation and angiogenesis protects neonatal mice from pressure overload-associated maladaptation
}

\author{
Mona Malek Mohammadi, ${ }^{1,2,3}$ Aya Abouissa, ${ }^{1}$ Isyatul Azizah, ${ }^{1}$ Yinuo Xie, ${ }^{1}$ Julio Cordero, ${ }^{4}$ \\ Amir Shirvani, ${ }^{2}$ Anna Gigina, ${ }^{2}$ Maren Engelhardt, ${ }^{5}$ Felix A. Trogisch, ${ }^{1,3}$ Robert Geffers, ${ }^{6}$ \\ Gergana Dobreva, ${ }^{3,4}$ Johann Bauersachs, ${ }^{2}$ and Joerg Heineke ${ }^{1,2,3}$ \\ 'Department of Cardiovascular Research, European Center for Angioscience, Medical Faculty Mannheim, Heidelberg \\ University, Mannheim, Germany. ${ }^{2}$ Department of Cardiology and Angiology, Hannover Medical School, Hannover, \\ Germany. ${ }^{3}$ German Center for Cardiovascular Research (DZHK) partner site Heidelberg/Mannheim, Heidelberg, Germany. \\ ${ }^{4}$ Department of Anatomy and Developmental Biology, Center for Biomedicine and Medical Technology Mannheim, \\ European Center for Angioscience, and 5 Institute for Neuroanatomy, Center for Biomedicine and Medical Technology \\ Mannheim, Medical Faculty Mannheim, Heidelberg University, Mannheim, Germany. ${ }^{6}$ Department Genome Analytics, \\ Helmholtz-Center for Infection Research GmbH, Braunschweig, Germany.
}

Cardiac pressure overload - for example, due to aortic stenosis - induces irreversible myocardial dysfunction, cardiomyocyte hypertrophy, and interstitial fibrosis in patients. In contrast with adult mice, neonatal mice can efficiently regenerate the heart after injury in the first week after birth. To decipher whether insufficient cardiac regeneration contributes to the progression of pressure overload-dependent disease, we established a transverse aortic constriction protocol in neonatal mice (nTAC). nTAC in the nonregenerative stage (at P7) induced cardiac dysfunction, myocardial fibrosis, and cardiomyocyte hypertrophy. In contrast, nTAC in the regenerative stage (at P1) largely prevented these maladaptive responses and was, in particular, associated with enhanced myocardial angiogenesis and increased cardiomyocyte proliferation, which both supported adaptation during nTAC. A comparative transcriptomic analysis between hearts after regenerative versus nonregenerative nTAC suggested the transcription factor CATA4 as master regulator of the regenerative gene program. Indeed, cardiomyocyte-specific deletion of GATA4 converted the regenerative $\mathrm{nTAC}$ into a nonregenerative, maladaptive response. Our new nTAC model can be used to identify mediators of adaptation during pressure overload and to discover potential therapeutic strategies.

Conflict of interest: The authors have declared that no conflict of interest exists.

Copyright: () 2019, American Society for Clinical Investigation.

Submitted: February 21, 2019

Accepted: July 16, 2019

Published: July 23, 2019

Reference information: /Cl Insight. 2019;4(16):e128336.

https://doi.org/10.1172/jci.

insight.128336.

\section{Introduction}

Pressure overload is a widespread deleterious stimulus imposed on the hearts of many adult patients suffering, for example, from chronic arterial hypertension or aortic valve stenosis $(1,2)$. The mouse model to induce transverse aortic constriction (TAC) in adult mice is widely used to understand the disease mechanisms of cardiac pressure overload (3). It typically triggers left ventricular hypertrophic remodeling that becomes progressively associated with cardiac dysfunction. At the cellular level, pressure overload entails cardiomyocyte hypertrophy, increasing interstitial fibrosis and capillary rarefaction during advanced stages $(1,2,4)$. Therapeutic relief from pressure overload (e.g., through medical treatment of arterial hypertension or transcatheter or surgical replacement of the aortic valve) halts disease progression and even leads to a partial recovery of cardiac function $(4,5)$. In advanced aortic valve stenosis, however, valve replacement no longer results in a normalization of heart function, likely due to cardiomyocyte degeneration and aggravated fibrosis $(4,6)$. Currently, mainly the maladaptive nature of hypertrophic remodeling is made responsible for this, although the irreversibility of cardiac damage suggests that insufficient myocardial regenerative capacity could also contribute to disease progression during pressure overload. 
Recent seminal research has revealed that, in contrast with the adult myocardium, neonatal mouse hearts retain some regenerative potential immediately after birth. In response to resection of the cardiac apex or to myocardial infarction on P1, the neonatal heart regrows functional myocardium within 21 days, virtually without scarring (7-9). Importantly, in response to LAD ligation, almost complete cardiac regeneration is observable as early as 7 days after injury (10). Neonatal heart regeneration is mediated mainly by proliferation of preexisting cardiomyocytes and is supported by angiogenesis, macrophage infiltration, and innervation $(8,9,11)$. Intriguingly, heart regeneration was reported in pigs and even in a human newborn after myocardial infarction (12-14). The neonatal cardiac regenerative capacity, however, is lost by P7 (7). Current research efforts aim to identify central regulators of neonatal heart regeneration that are differentially expressed after injury between $\mathrm{P} 1$ and $\mathrm{P} 7$, in order to design potentially novel therapeutic strategies for promoting cardiac restoration after myocardial infarction. Accordingly, we recently demonstrated a high myocardial expression of the transcription factor GATA4 shortly after birth at P1, which strongly diminishes until P7 (15). GATA4 belongs to the GATA family of transcription factors, which all share a conserved 2 zinc finger-containing DNA binding domain and bind to the DNA motif (A/T)GATA(A/G) (16). In mice, cardiomyocyte GATA4 promotes intrauterine cardiac development, in part by driving cardiomyocyte proliferation; however, in the adult, GATA4 triggers cardiac hypertrophy, cell survival, and angiogenesis during pressure overload (17-19). As demonstrated by us and others, the high cardiac levels of GATA4 shortly after birth are necessary for neonatal mouse heart regeneration after myocardial injury at P1, as well as for zebrafish cardiac regeneration, because GATA4 promotes cardiomyocyte proliferation and capillary growth $(15,20,21)$.

To test whether adaptation to cardiac pressure overload relies on regenerative mechanisms in the myocardium, we established for the first time to our knowledge a TAC protocol in neonatal mice (nTAC). Comparison of the neonatal response to nTAC during the regenerative phase (surgery at P1) versus the nonregenerative phase (surgery at P7) revealed that, in the regenerative phase, nTAC induces a pure adaptive response with preserved cardiac function and increased cardiomyocyte proliferation and with growth of myocardial nerves and angiogenesis, but not with fibrosis. In contrast, nTAC in the nonregenerative phase triggered cardiac dysfunction, cardiomyocyte hypertrophy, and interstitial myocardial fibrosis but not cardiomyocyte proliferation, angiogenesis, or innervation. Transcriptomic profiling in conjunction with nTAC experiments in cardiomyocyte-specific GATA4 KO mice revealed GATA4 as a master regulator of the adaptive response to pathological pressure overload in the neonatal, regenerative phase. Hence, the adaptive, regenerative response toward pressure overload immediately after birth could serve as a blueprint on how to therapeutically modify the adult heart's response to pressure overload to counteract the development of heart failure in the future.

\section{Results}

Functional adaptation to pressure overload after nTAC surgery at P1 but not after nTAC at P7. In order to compare the myocardial response to pressure overload in the regenerative versus the nonregenerative phase in neonatal mice, nTAC was induced for 2 weeks in 1-day-old mice (14dp1dTAC, regenerative phase, blue arrow in Figure 1A) or for 1 week in 7-day-old mice (7dp7dTAC, nonregenerative phase, red arrow) (Figure 1A). This approach ensured that, for both groups, the experiment ended at P14 and that, therefore, a similar degree of postnatal development, body size, and aortic constriction could be assumed. In a third group of mice, a sham operation was conducted at P1, and the mice were sacrificed at P14. Induction of pressure overload in 1-day-old mice was confirmed at $\mathrm{P} 2$ by an increased flow velocity ratio of the right to left common carotid artery (RCA/LCA) (Supplemental Figure 1A; supplemental material available online with this article; https://doi.org/10.1172/jci.insight.128336DS1). Measuring this flow velocity ratio also revealed a similar degree of left ventricular pressure overload 7 days after TAC at P1 (7dp1dTAC) compared with immediately after TAC at day $7(0 \mathrm{dp} 7 \mathrm{dTAC})$ as well as before sacrifice in 14dp1dTAC and 7dp7dTAC mice (Supplemental Figure 1B and Figure 1B). At the end of the study (P14), the heart weight to body weight (HW/BW) ratio was significantly increased in both 14dp1dTAC and 7dp7dTAC mice compared with sham (Figure 1C). Interestingly, pulmonary congestion (i.e., increased lung weight to body weight ratio [LW/BW]) was only observed in 7dp7dTAC mice (Figure 1C). The left ventricular ejection fraction (EF) was assessed by echocardiography and was reduced in 7dp7dTAC, but not in 14dp1dTAC mice compared with sham-operated mice. Wall thickness and left ventricular end-diastolic area (LVEDA) significantly increased in 14dp1dTAC - but not in 7dp7dTAC mice (Figure 1D). The HW/BW ratio 
A

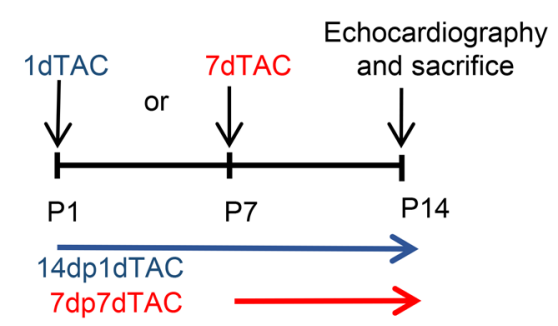

C

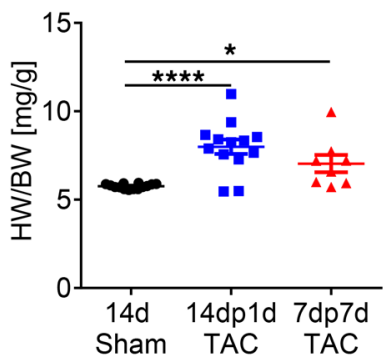

D

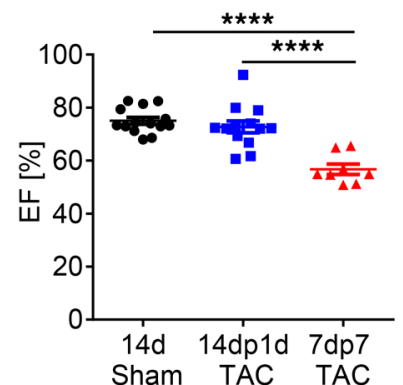

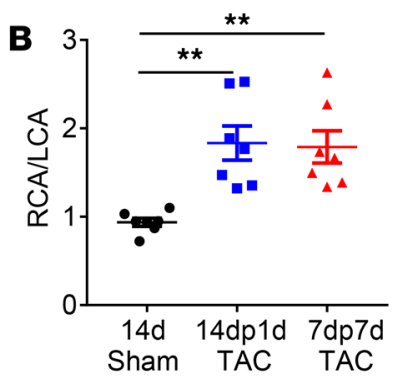
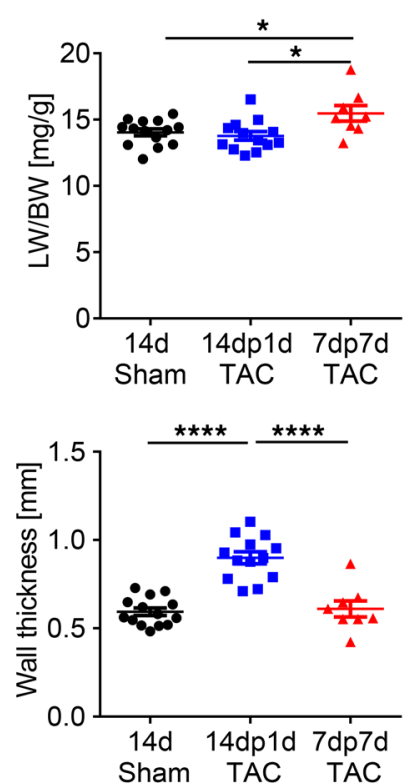

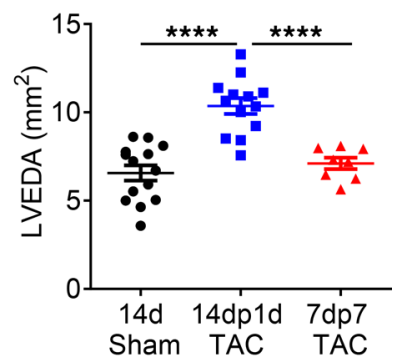

Figure 1. Pressure overload reduces cardiac function in 7-day-old mice but not in 1-day-old mice. (A) A schematic representation of the time line of nTAC in the regenerative ( $\mathrm{P} 1$, named 14dp1dTAC) and nonregenerative ( $\mathrm{P7}$, named 7dp7dTAC) phases. (B) Flow velocity ratio of the right common carotid artery (RCA) to left common carotid artery (LCA) in 14-day sham, 14dp1dTAC, and 7dp7dTAC mice; $n=7$ for each group. ** $P<0.005$ (1-way ANOVA/Sidak's multiple comparisons test). (C) Heart weight to body weight (HW/BW) and lung weight to body weight (LW/BW) ratio in sham, 14dp1dTAC, and 7dp7dTAC mice; $n=14$, sham; $n=13,14 \mathrm{dp1dTAC} ; n=8$, 7dp7dTAC. ${ }^{*} P<0.05$, ${ }^{* * * *} P<0.0001$ (1-way ANOVA/Sidak's multiple comparisons test). (D) Ejection fraction (EF), wall thickness, and left ventricular end-diastolic area (LVEDA) assessed by echocardiography; $n=14$, sham; $n=13,14 \mathrm{dp} 1 \mathrm{dTAC} ; n=8,7 \mathrm{dp} 7 \mathrm{dTAC}$ ). ${ }^{* * *} P<$ 0.0001 (1-way ANOVA/Sidak's multiple comparisons test).

increased as soon as 7 days after surgery in 1-day-old mice (7dp1dTAC), but no increase in LW/BW was observed compared with 7-day-old sham mice (Supplemental Figure 1C). EF and LVEDA were also not changed at this early time point, but the wall thickness was increased versus sham-operated mice 7 days after nTAC at P1 (Supplemental Figure 1D).

While the RCA/LCA flow velocity ratio was increasing with age as the aortic ligature got tighter due to organismal growth, it did not differ at P21 between mice 21 days after TAC at P1 (21dp1dTAC) and 14 days after TAC at P7 (14dp7dTAC) (Supplemental Figure 1, E and F). The HW/BW ratio similarly increased in both groups, but signs of pulmonary congestion were only found in 14dp7dTAC mice (Supplemental Figure 1G). The EF remained reduced after 14 days of TAC at P7 (14dp7dTAC) compared with sham, but this was not the case 21 days after TAC at P1 (Supplemental Figure 1H). The LVEDA was not increased in any of the groups compared with sham; however, wall thickness was increased in $21 \mathrm{dp} 1 \mathrm{dTAC}$ but not in 14dp1dTAC (Supplemental Figure 1H). Therefore, nTAC at P1 in the regenerative phase - remained in a compensated, adaptive stage for 21 days, while its induction at $\mathrm{P} 7$ produces a maladaptive phenotype for 7 as well as for 14 days.

Pressure overload triggered cardiomyocyte proliferation and angiogenesis in 1-day-old mice. Histological analysis using Sirius Red staining and quantification of fibrotic areas in heart sections revealed a significantly increased amount of interstitial fibrosis in hearts of 7dp7dTAC mice compared with sham and 14dp1dTAC mice (Figure 2A). A significant amount of fibrosis was also observed in 14dp7dTAC, while it was not in 7dp1dTAC nor in 21dp1dTAC mice (Supplemental Figure 2, A, B, D, and E). To quantify 
A

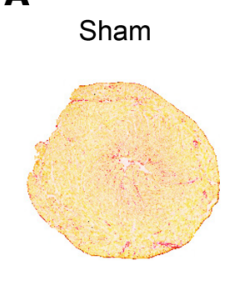

B
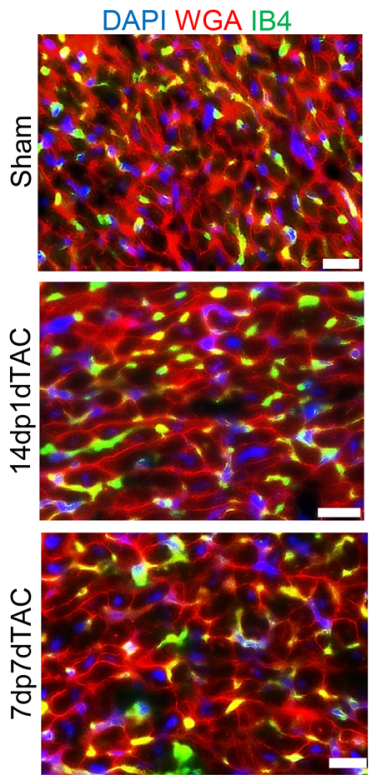

14dp1dTAC
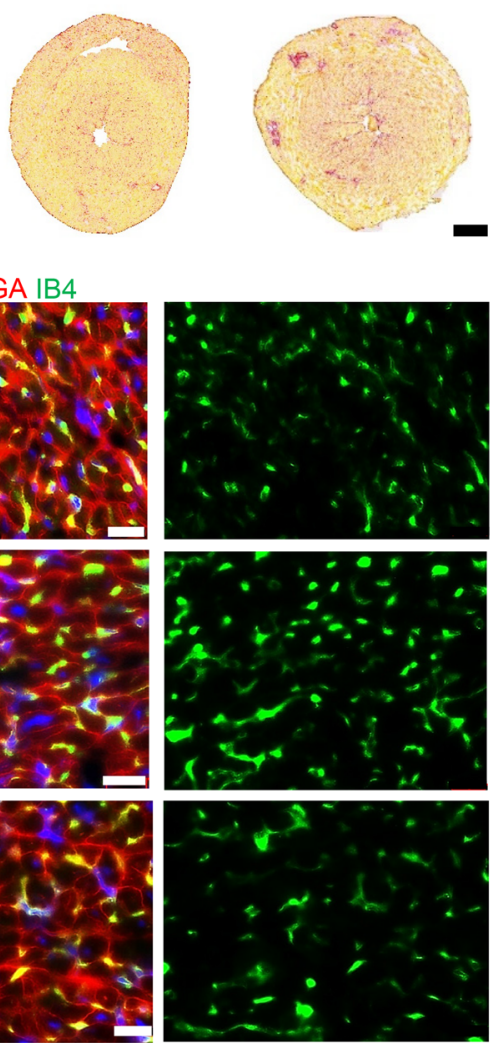
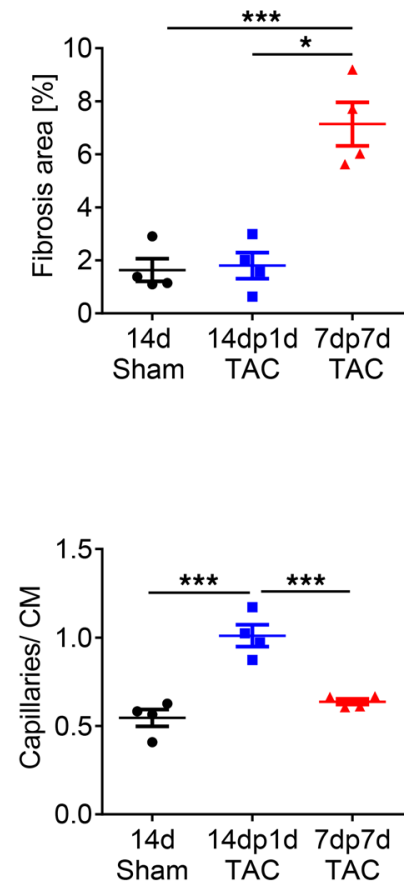

Figure 2. Pressure overload induces angiogenesis but not fibrosis in 1-day-old mice. (A) Sirius Red staining of heart sections of the indicated mice to detect fibrosis and its quantification. Scale bar: $500 \mu \mathrm{m} ; n=10$, sham; $n=7,14 \mathrm{dp} 1 \mathrm{~d}$ TAC; $n=8,7 \mathrm{dp7dTAC.}{ }^{*} P<0.05,{ }^{* * *} P<0.001$ (Kruskal Wallis test/Dunn's multiple comparisons test). (B) Immunofluorescence staining of heart sections for isolectin B4 (IB4), WCA, and DAPI and quantification of the capillaries/cardiomyocyte (CM) ratio. Scale bar: $20 \mu \mathrm{m} ; n=4$, 14d sham; 14dp1dTAC and 7dp7dTAC. ${ }^{* * *} P<0.001$ (1-way ANOVA/Sidak's multiple comparisons test).

the regenerative activity in the myocardium, immunofluorescence staining for endothelial cells (with endothelial cell-specific Isolectin B4 [IB4]), cardiomyocyte proliferation (Ki67), mitosis (phosphorylated-histone $\mathrm{H} 3$ [pH3]), and cytokinesis (Aurora B kinase staining of the midbody during cell division) together with staining for Troponin T, Nkx2.5, or WGA to identify cardiomyocytes - was performed. We observed an increased capillary density, as well as enhanced cardiomyocyte mitosis and cytokinesis, only after nTAC in the regenerative phase (at P1) in the 7dp1dTAC, 14dp1dTAC, and 21dp1dTAC experimental groups (Figure 2B; Figure 3, A-D, F, and G; Supplemental Figure 2, C and F; and Supplemental Figure 3, A and C). Cardiac hypertrophy (measured as cardiomyocyte cross-sectional area) was transiently increased in $7 \mathrm{dp} 1 \mathrm{dTAC}$, but not in $14 \mathrm{dp} 1 \mathrm{dTAC}$ or $21 \mathrm{dp} 1 \mathrm{dTAC}$ mice, compared with sham mice (Figure 3E and Supplemental Figure 3, B, D, and E). In contrast, we found a significant and sustained increase of cardiomyocyte hypertrophy in mice from the 7dp7dTAC and 14dp7dTAC groups (Figure 3E and Supplemental Figure 3, B, D, and E). Taken together, nTAC, initiated at P1 in the regenerative phase (7dp1dTAC, 14dp1dTAC, $21 \mathrm{dp} 1 \mathrm{dTAC}$ ) resulted in a compensated cardiac function and was associated with cardiomyocyte proliferation and angiogenesis but not with fibrosis at different time points. In contrast, when nTAC was started at P7 (i.e., in the nonregenerative phase, 7dp7dTAC, 14dp7dTAC), it led to cardiac dysfunction, sustained hypertrophy, and fibrosis.

Rapamycin treatment ablated the regenerative response to $n T A C$ at $P 1$. We next wanted to decipher the functional importance of enhanced cardiomyocyte proliferation for cardiac adaptation during regenerative nTAC. To this end, we aimed to induce regenerative TAC and treated the mice with the macrolide antibiotic rapamycin, which acts as an effective inhibitor of cardiomyocyte proliferation (22). Indeed, treatment 
A

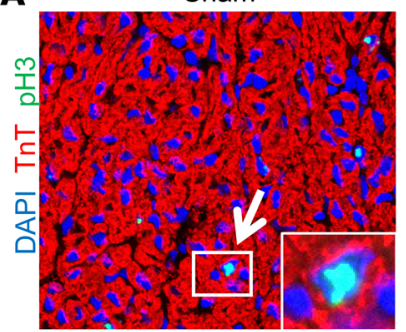

C

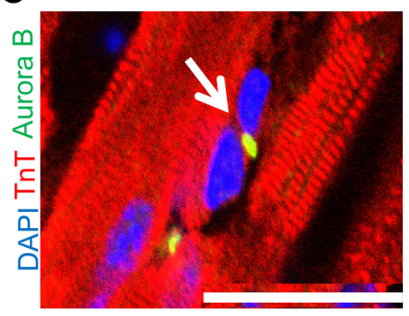

$\mathbf{F}$

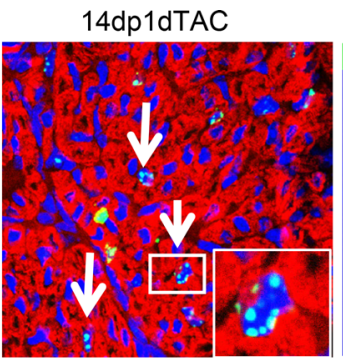

D

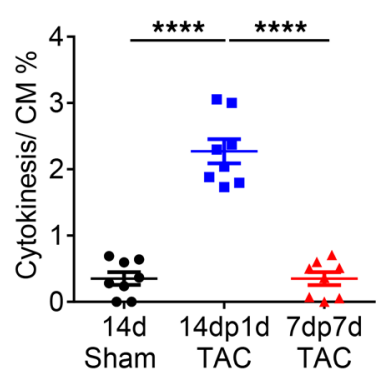

7dp7dTAC

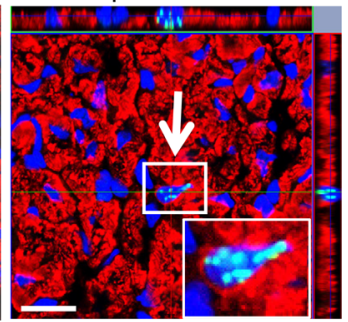

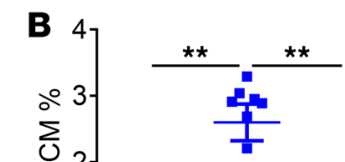

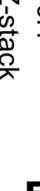

¿
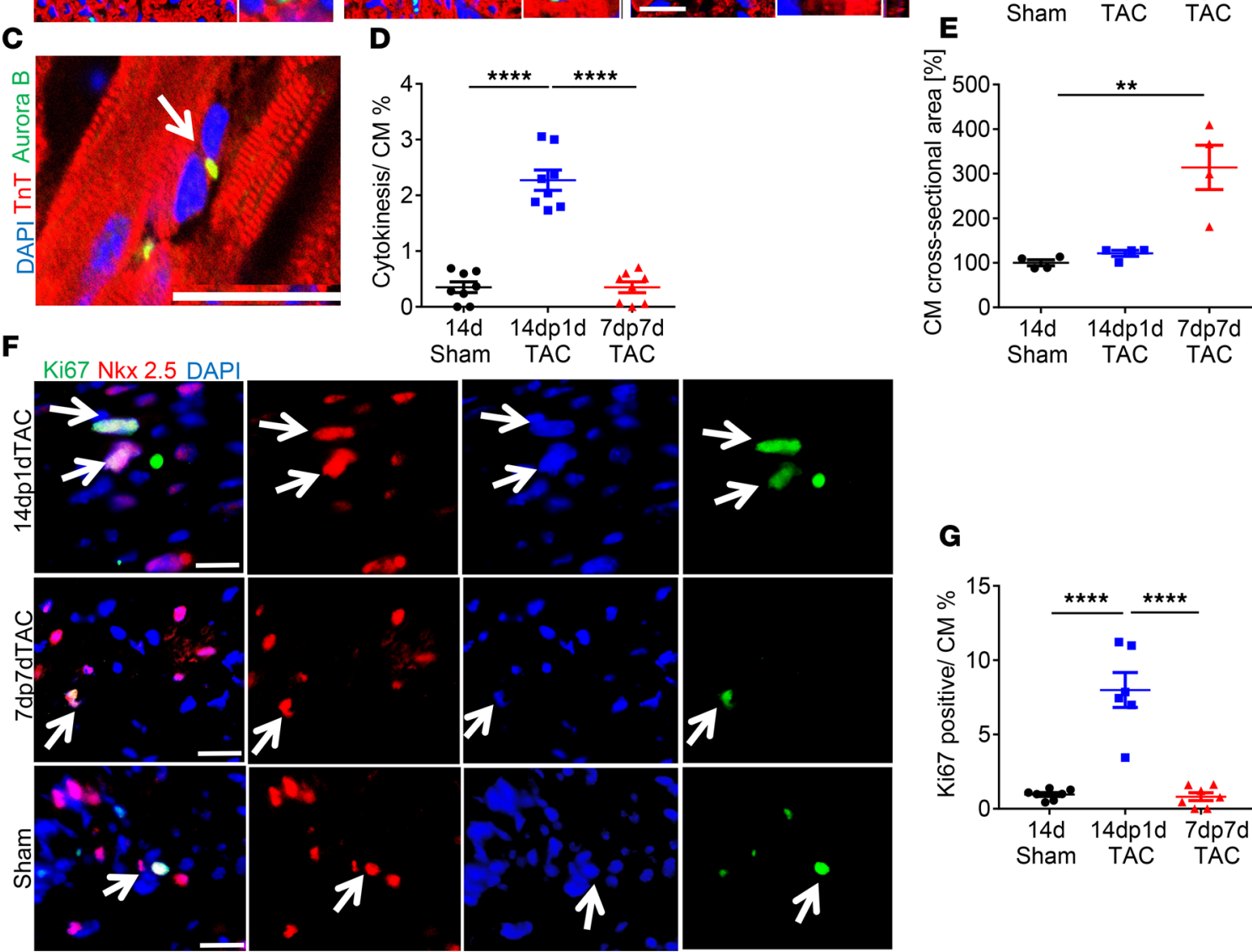

\section{G}

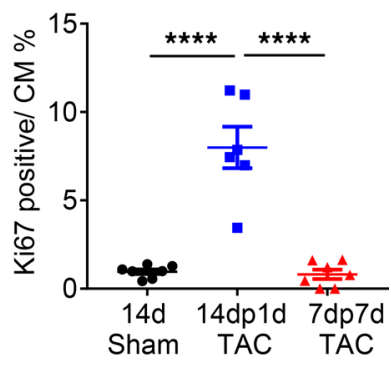

Figure 3. Pressure overload triggers cardiomyocyte proliferation in 1-day-old but not 7-day-old mice. (A) Immunofluorescence staining of heart sections for phospho-Histone $3(\mathrm{pH} 3)$, Troponin T, and DAPI. Arrows indicate $\mathrm{pH} 3$-positive cardiomyocytes. Inserts represent higher magnification of pH3 positive cardiomyocytes. The panel on the right and above 7dp7dTAC shows a Z-stack view of the detected pH3-positive cardiomyocyte. Scale bar: $20 \mu \mathrm{m}$. (B) Quantification of pH3-positive cardiomyocytes; $n=8$ for each condition. ${ }^{* *} P<0.005$ (Kruskal Wallis test/Dunn's multiple comparisons test). (C and $\mathbf{D}$ ) Immunofluorescence staining for Aurora B, Troponin T, and DAPI (arrow indicates cytokinesis) and quantification of cardiomyocyte cytokinesis (Aurora B positivity at the midbody of dividing cardiomyocytes) per total cardiomyocytes. Scale bar: $20 \mu \mathrm{m} ; n=8$ for each condition. ${ }^{* * * *} P<0.0001$ (1-way ANOVA/ Sidak's multiple comparisons test). (E) Cardiomyocyte cross-sectional area in the indicated mice; $n=4$ for each condition. ${ }^{* *} P<0.005$ (Kruskal Wallis test/Dunn's multiple comparisons test). (F) Immunofluorescence staining of heart sections for Ki67, Nkx2.5, and DAPI. Scale bar: $20 \mu \mathrm{m}$. Arrows indicate nuclei positive for Ki67, Nkx2.5, and DAPI. (C) Quantification of Ki67-positive cardiomyocytes; $n=7$ for 14-day sham and 7dp7dTAC; arrows indicate Ki67-positive cardiomyocytes; $n=6$ for 14dp1dTAC. ${ }^{* * *} P<0.0001$ (1-way ANOVA/Sidak's multiple comparisons test).

of isolated neonatal rat cardiomyocytes with rapamycin in vitro strongly decreased cardiomyocyte proliferation (assessed by pH3 and Aurora B midbody staining) (Figure 4, A and B). We administered rapamycin or the solvent as a control to mice daily for 7 days after nTAC on P1 (Figure 4C). Intriguingly, rapamycin markedly increased mortality in mice after nTAC but not after sham surgery (Figure 4D). Analyzing the hearts of the surviving mice after 1 week of pressure overload revealed an increase in HW/BW after nTAC in control mice but not in rapamycin-treated mice (Figure 4E). Echocardiography showed no difference in the EF of the surviving mice after nTAC (Figure 4F). Quantification of myocardial fibrosis revealed a nonsignificantly exaggerated fibrotic response in the hearts of rapamycin-treated mice after nTAC (Figure 5A). Unexpectedly, the capillary density was reduced by rapamycin after sham and nTAC surgery (Figure 5, B and C). Cardiomyocyte mitosis ( $\mathrm{pH} 3$ ) and cytokinesis (Aurora B midbody staining) increased due to nTAC 
A
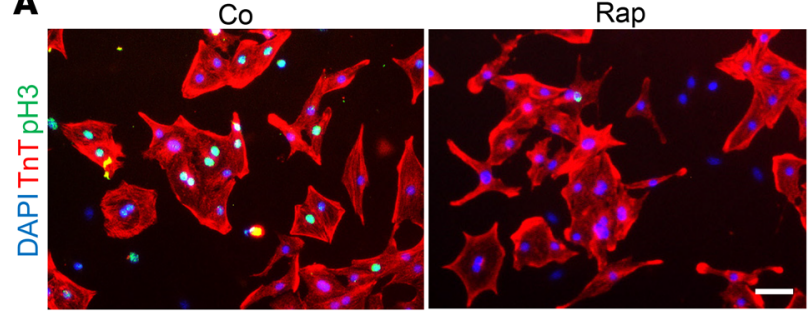

C

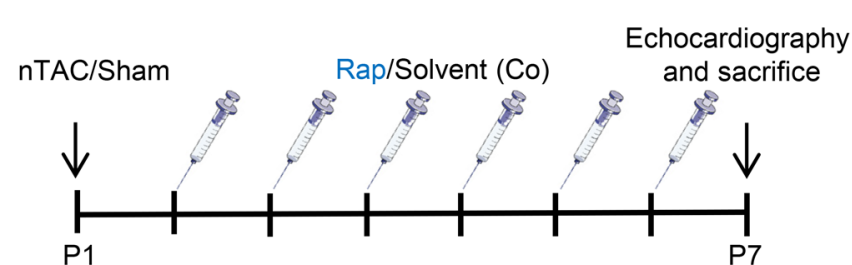

E

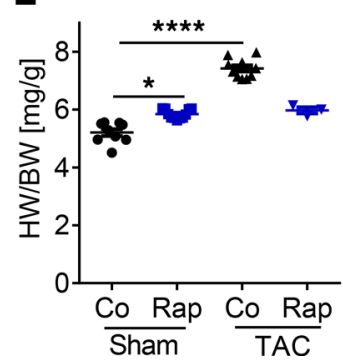

F

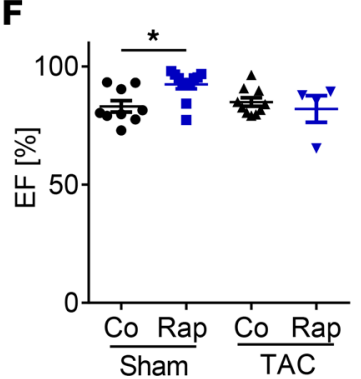

B
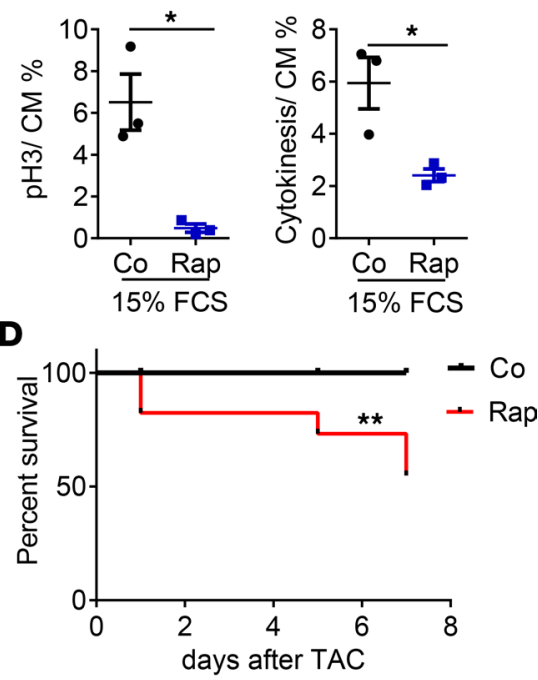

Figure 4. Increased mortality during rapamycin treatment after nTAC in the regenerative phase. (A) Immunostaining of neonatal cardiomyocytes for pH3, Troponin T, and DAPI after treatment with solvent (control, Co) or rapamycin (Rap). Scale bar: $50 \mu \mathrm{m}$. (B) Quantification of mitosis and cytokinesis in Rap- and Co-treated (solvent) isolated neonatal cardiomyocytes in vitro; $n=3$ for each condition. ${ }^{*} P<0.05$ (unpaired $t$ test). (C) A schematic representation of the experimental time schedule. (D) Kaplan-Meier survival curve of Rap- and Co-treated mice after nTAC; $n=10$, Co; $n=8$, Rap. ${ }^{* *} P<0.005$ (Cehan-Breslow-Wilcoxon test). (E) HW/BW ratio of the indicated mice after sham and nTAC surgery; $n=9$, Co sham; $n=11$, Rap sham; $n=10$, Co TAC; $n=3$, Rap TAC. ${ }^{*} P<0.05$, ${ }^{* * * *} P<$ 0.0001 (Kruskal Wallis test/Dunn's multiple comparisons test). (F) Echocardiographic left ventricular ejection fraction (EF) of mice treated as indicated; $n=9$, Co sham; $n=11$, Rap sham; $n=10$, Co TAC; $n=3$, Rap TAC. ${ }^{*} P<0.05$ (Kruskal Wallis test/Dunn's multiple comparisons test).

but were potently reduced by rapamycin in sham and nTAC mice (Figure 5, D and E). Cardiomyocyte cross-sectional area as an indicator of hypertrophy slightly increased 7 days after nTAC in the regenerative phase but was also nonsignificantly reduced by rapamycin administration (Figure $5 \mathrm{~F}$ ). Collectively, rapamycin treatment entailed reduced cardiomyocyte proliferation, but it also reduced angiogenesis after nTAC during the regenerative phase and thereby triggered mouse mortality and cardiac fibrosis.

Pharmacological inhibition of angiogenesis blocked adaptation during regenerative neonatal TAC. Given the increased angiogenesis in regenerative nTAC (e.g., in 14dp1dTAC mice), and the concomitant reduction of cardiomyocyte proliferation and angiogenesis due to rapamycin treatment, we wanted to decipher the selective impact of angiogenesis for the adaptive response to nTAC in the regenerative phase. Therefore, nTAC or sham surgery were performed on P1 and an angiogenesis inhibitor (PTK787, which inhibits VEGFR2 downstream signaling in endothelial cells) or DMSO were injected daily starting from the 7th day after surgery (Figure 6A). While we did not observe any mortality during the first 7 days after nTAC (before administration of PTK787), mice started to die very soon after the administration of PTK787. Thus, we terminated the study after only 3 injections on day 10 after nTAC (Figure 6B).

The HW/BW ratio increased in both treated and control mice after nTAC compared with their corresponding sham mice, but there was no difference between treated and control nTAC mice (Figure 6C). There was also no difference in the EF of control and the surviving PTK787-treated mice afternTAC (Figure 6D). The antiangiogenesis treatment resulted in a considerable amount of fibrosis after nTAC compared with control nTAC mice (Figure 6, E and F), and - as expected - quantification of myocardial capillary density revealed a strong reduction of angiogenesis in the PTK787- compared with the DMSO-treated (control) mice after nTAC (Figure 7, A and B). Interestingly, administration of PTK787 also inhibited cardiomyocyte proliferation 
A
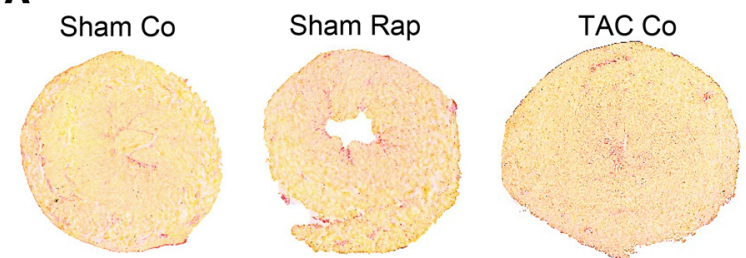

B
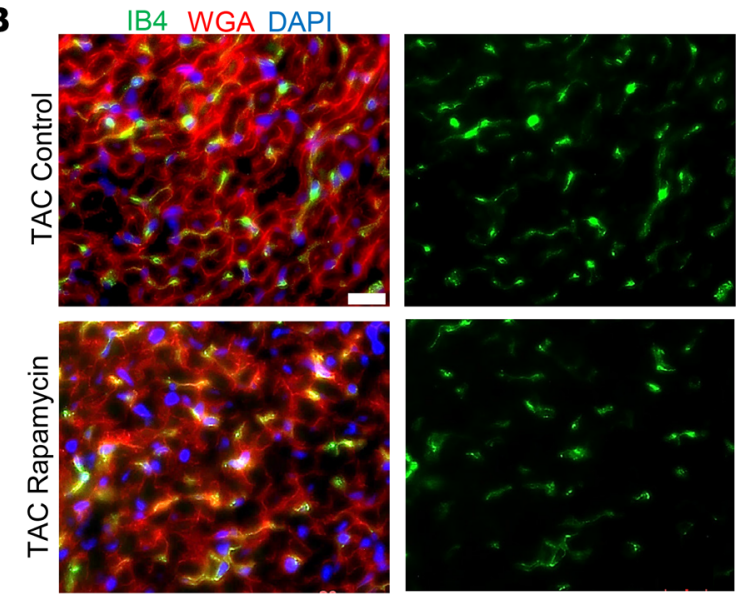

D

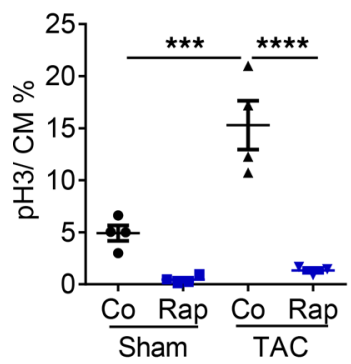

E

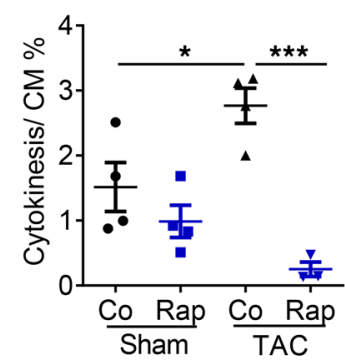

TAC Rap
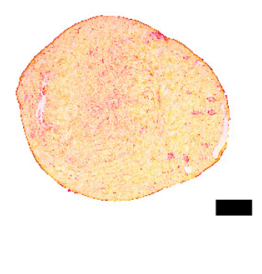

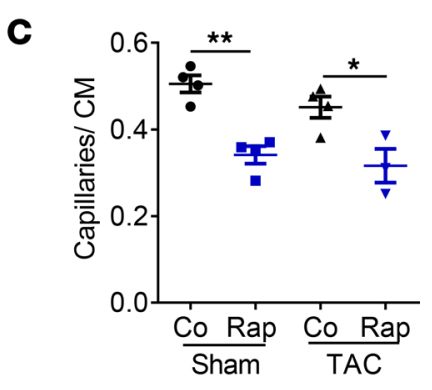

$\mathbf{F}$

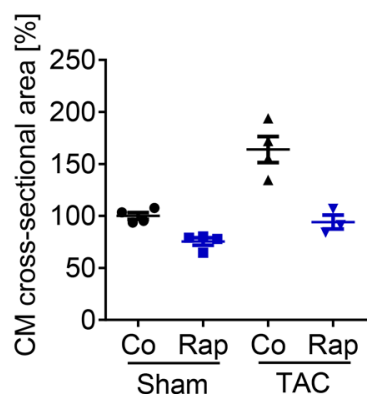

Figure 5. Increased fibrosis following pharmacological inhibition of cardiomyocyte proliferation and angiogenesis after nTAC in the regenerative phase. (A) Representative images and quantification of fibrosis in the hearts after Sirius Red staining of the indicated mice. $n=9$, Co sham; $n=11$, Rap sham; $n=10$, Co TAC; $n=3$ Rap TAC; Kruskal Wallis test/Dunn's multiple comparisons test. Scale bar: $20 \mu \mathrm{m}$. (B) Immunofluorescence staining of the heart sections for IB4, WGA, and DAPI. Scale bar: $20 \mu \mathrm{m}$. (C) Quantification of the capillaries/cardiomyocyte (CM) ratio; $n$ $=4$, Co sham; $n=4$, Rap sham; $n=4$, Co TAC; $n=3$, Rap TAC. ${ }^{*} P<0.05$, ${ }^{*} P<0.005$ (1-way ANOVA/Sidak's multiple comparisons test). ( $\mathbf{D}$ and $\mathbf{E}$ ) Quantification of pH3-positive cardiomyocytes (D) and of cardiomyocytes undergoing cytokinesis (E); $n=4$, Co sham; $n=4$, Rap sham; $n=4$, Co TAC; $n=3$, Rap TAC. ${ }^{*} P<0.05$, ${ }^{* * *} P<0.001$, ${ }^{* * *} P<$ 0.0001 (1-way ANOVA/Sidak's multiple comparisons test). (F) Cardiomyocyte cross-sectional area in the myocardium of mice as indicated; $n=4$, Co sham; $n=4$, Rap sham; $n=4$, Co TAC; $n=3$, Rap TAC. Kruskal Wallis test/Dunn's multiple comparisons test.

(mitosis and cytokinesis) after nTAC versus DMSO treatment (Figure 7, C and D). Unexpectedly, we also detected a significantly reduced level of cardiomyocyte mitotic activity (pH3 staining) in the DMSO treatment group 10 days after nTAC versus sham, which is in contrast with the increased cardiomyocyte mitosis that we detected 7 and 14 days after nTAC at P1. While we cannot fully explain this discrepancy, we assumed that DMSO treatment after nTAC might at least be partially responsible for this phenomenon (23). nTAC induced cardiomyocyte hypertrophy (measured by cardiomyocyte cross-sectional area) in PTK787-treated compared with control nTAC mice (Figure 7E). To exclude a direct effect of PTK787 on cardiomyocytes, we treated neonatal rat cardiomyocytes with PTK787 or DMSO. This experiment showed that PTK787 had no direct effect on cardiomyocyte proliferation, cytokinesis, or hypertrophy in the presence of $15 \%$ FCS (Figure $7, \mathrm{~F}$ and $\mathrm{G}$ ). Our data suggest that induction of angiogenesis in 1-day-old mice might be a prerequisite for the induction of cardiomyocyte proliferation and cardiac adaptation during pressure overload. 
A

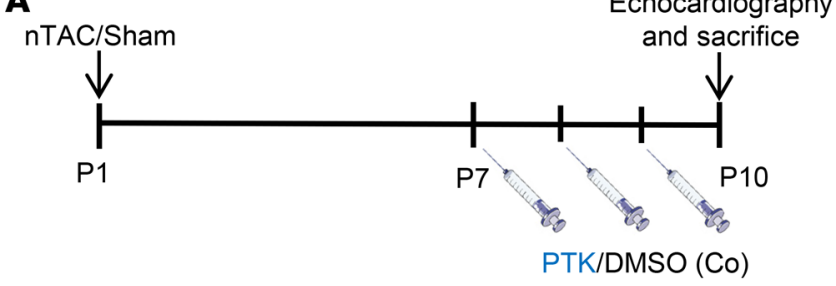

B

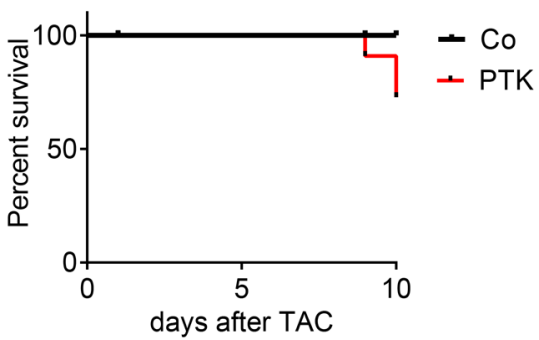

C

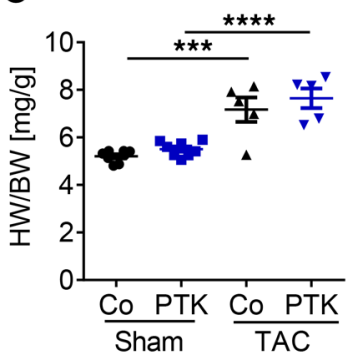

E
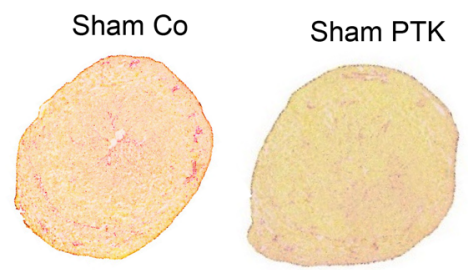

TAC Co

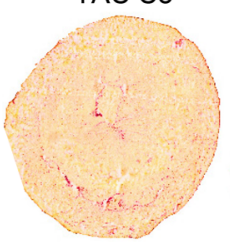

TAC PTK

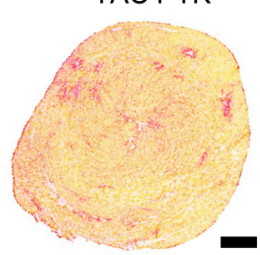

D

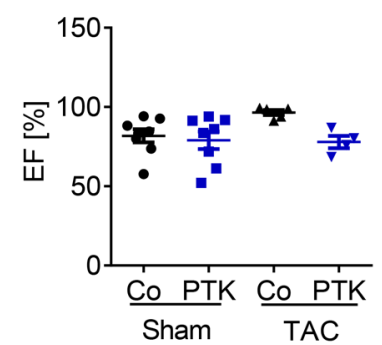

$\mathbf{F}$

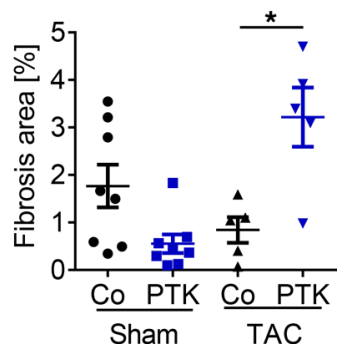

Figure 6. Augmented fibrosis following pharmacological inhibition of angiogenesis after nTAC in neonatal mice. (A) A schematic representation of the experimental time schedule. (B) Kaplan-Meier survival curve of PTK- and control-treated (Co-treated) mice after nTAC. $n=10$, Co; $n=8$, PTK. (C) HW/BW ratio in PTK or Co sham- and TAC-operated mice; $n=8$, Con sham; $n=8$ PTK sham; $n=5$, Con TAC; $n=5$, PTK TAC. ${ }^{* *} P<0.001,{ }^{* * * *} P<0.0001$ (1-way ANOVA/Sidak's multiple comparisons test). (D) Echocardiographic left ventricular ejection fraction (EF) of Co- and PTK-treated sham and nTAC mice; $n=8$, Con sham; $n=8$, PTK sham, $n=5$, Con TAC; $n=5$, PTK TAC. (E and F) Sirius Red staining of heart sections from the indicated mice (scale bar: $500 \mu \mathrm{m}$ ) (E) and quantification of myocardial fibrosis (F); $n=8$, Con sham; $n=8$, PTK sham; $n=5$, Con TAC; $n=5$, PTK TAC. ${ }^{*} P<0.05$ (Kruskal Wallis test/Dunn's multiple comparisons test). PTK, PTK787.

One-day-old mice upregulated a proregenerative gene expression pattern in response to nTAC. To gain more insight into the mechanisms that might be responsible for better cardiac adaptation and regeneration of 1-day- but not 7-day-old mice in response to pressure overload (nTAC), we performed a comprehensive transcriptomic analysis of the left ventricle from 14dp1dTAC (regenerative), 7dp7dTAC (nonregenerative), and 14-day-old sham hearts by RNA sequencing (RNA-seq). The heatmap of the differentially regulated genes (significantly changed in expression more than 1.5 -fold, $P<0.05$ ) among $14 \mathrm{dp} 1 \mathrm{dTAC}$, 7dp7dTAC, and sham is illustrated in Figure 8 together with the main gene ontology (GO) terms (biological processes) observed in the 5 clusters of coregulated genes. Direct comparison of cardiac gene expression profiles during nTAC in the regenerative versus the nonregenerative phase (14dp1dTAC versus 7dp7dTAC) revealed many differentially expressed genes between these groups (Figure 9). Significantly upregulated genes in the regenerative 14dp1dTAC compared with nonregenerative 7dp7dTAC mainly belonged to the GO categories immune response, positive regulation of cell proliferation, angiogenesis, muscle contraction, wound healing, and, initially surprising to us, positive regulation of axonogenesis. On the other hand, the downregulated genes in regenerative 14dp1dTAC mainly belonged to GO categories negative regulation of cell proliferation and positive regulation of apoptosis (Figure 9). Example genes for each category are included in Figure 9. With regard to the embryonic genes that are typically reexpressed in the left ventricle of adult mice during cardiac overload, we found similar upregulation of ANP (Nppa) and BNP (Nppb) mRNAs in regenerative (14dp1dTAC) and in the nonregenerative (7dp7dTAC) mice, 
A
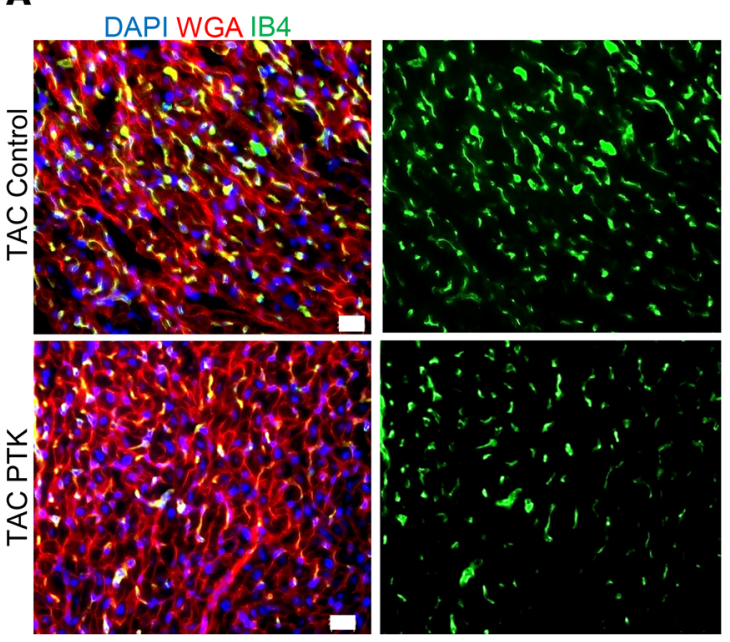

F
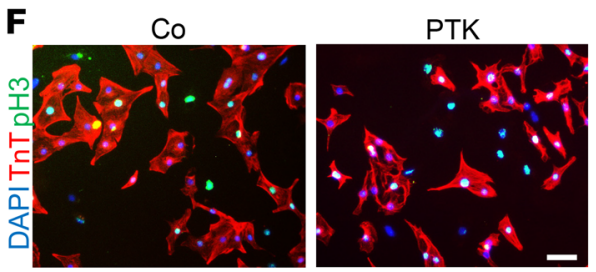

B

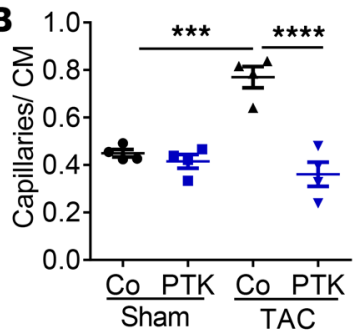

D

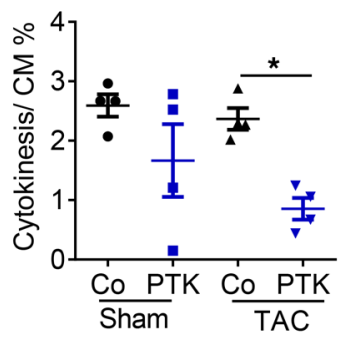

G
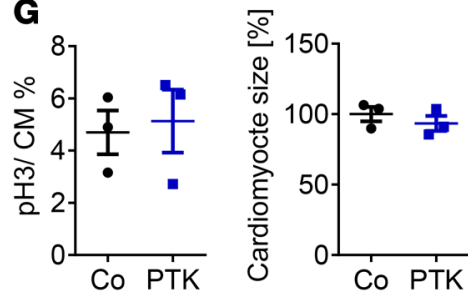
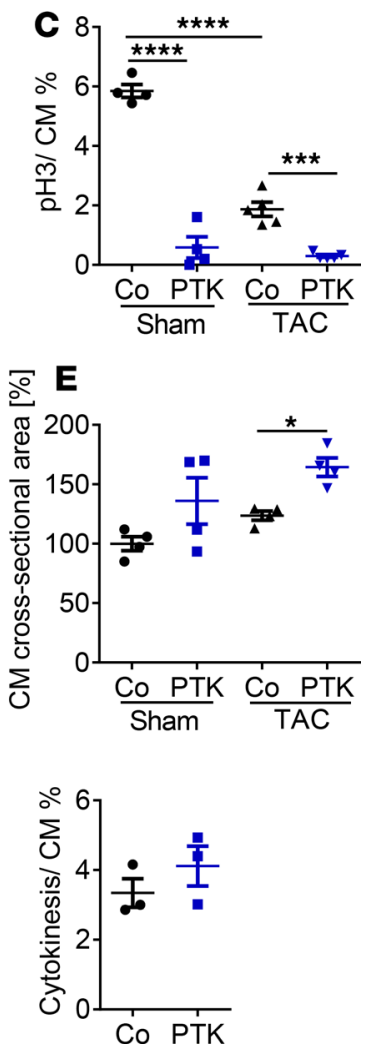

Figure 7. Diminished cardiomyocyte proliferation following pharmacological inhibition of angiogenesis after $\mathrm{nTAC}$ in neonatal mice. (A and B) Immunofluorescence staining of heart sections for IB4, WCA, and DAPI (scale bar: $20 \mu \mathrm{m}$ ) (A) and quantification of capillaries/cardiomyocyte (B); $n=4$ per group. ${ }^{* * *} P<0.001,{ }^{* * *} P<0.0001$ (1-way ANOVA/Sidak's multiple comparisons test). (C and D) Quantification of pH3-positive cardiomyocytes (C) and cardiomyocyte cytokinesis rates (D) per total cardiomyocytes in the indicated mice; $n=4$ per group. ${ }^{*} P<0.05,{ }^{* *} P<0.001,{ }^{* * * *} P<0.0001$ (1-way ANOVA/multiple comparison, Sidak's multiple comparisons test). (E) Cardiomyocyte (CM) cross-sectional area in the indicated mice; $n=4$ per group. ${ }^{*} P<0.05$ (1-way ANOVA/Sidak's multiple comparisons test). (F) Immunofluorescence staining of neonatal cardiomyocytes for pH3, Troponin T, and DAPI after treatment with DMSO (Co) and PTK787 in vitro. Scale bar: $50 \mu \mathrm{m}$. (G) Quantification of mitosis, cytokinesis, and cardiomyocyte size in isolated neonatal cardiomyocytes treated with PTK or Co in vitro; $n=3$ per group (unpaired $t$ test). PTK, PTK787; Co, control.

but $\beta$-MHC (Myh7) was only upregulated after nonregenerative nTAC, while $\alpha$-MHC (Myhø) expression remained unchanged (Supplemental Figure 4, A-D). All of the genes in the different GO categories and their dependence on GATA4 binding are shown in Supplemental Figure 5.

Because we observed many upregulated axonogenesis genes in 14dp1dTAC compared with 7dp7dTAC, we assessed nerve growth by immunostaining for the neurite marker TBB3 (class III $\beta$-tubulin) in heart sections of 14dp1dTAC and 7dp7dTAC mice, which revealed hyperinnervation in the hearts after nTAC in the regenerative compared with the nonregenerative phase (Figure 10A). Similarly, because we found increased expression of inflammatory genes in the left ventricles of 14dp1dTAC mice, we quantified the abundance of leukocytes (CD45-positive) in the myocardium and found more leukocytes in hearts after regenerative 14dp1dTAC versus nonregenerative 7dp7dTAC (Figure 10B). Hence, neonatal mice, immediately after birth, induce a regenerative, adaptive gene expression pattern in response to pressure overload that promotes cardiomyocyte proliferation as well as myocardial inflammatory cell recruitment, angiogenesis, and nerve growth.

The majority of upregulated genes in regenerative 14dp1dTAC are directly bound by GATA4. Since we have previously shown that GATA4 is highly expressed in 1-day-old but dramatically reduced in 7-day-old mouse hearts, we investigated whether GATA4 was a direct modulator of the upregulated cardiac genes in $14 \mathrm{dp} 1 \mathrm{dTAC}$ versus $7 \mathrm{dp} 7 \mathrm{dTAC}$. Crossing the upregulated genes in the regenerative nTAC with previously published cardiac GATA4 ChIP-sequencing (ChIP-seq) data (NCBI's Gene Expression Omnibus database, GSE52123) revealed that 74\% (696 genes) of the upregulated genes in 14dp1dTAC are directly bound by GATA4 (Figure 11A and ref. 24). Perhaps not surprisingly, these GATA4-bound genes belonged to similar GO classes (immune system, proliferation, angiogenesis, neuron, wound healing, and muscle contraction genes) as observed for all 14dp1dTAC upregulated genes (Supplemental Figure 5). To visualize the DNA 


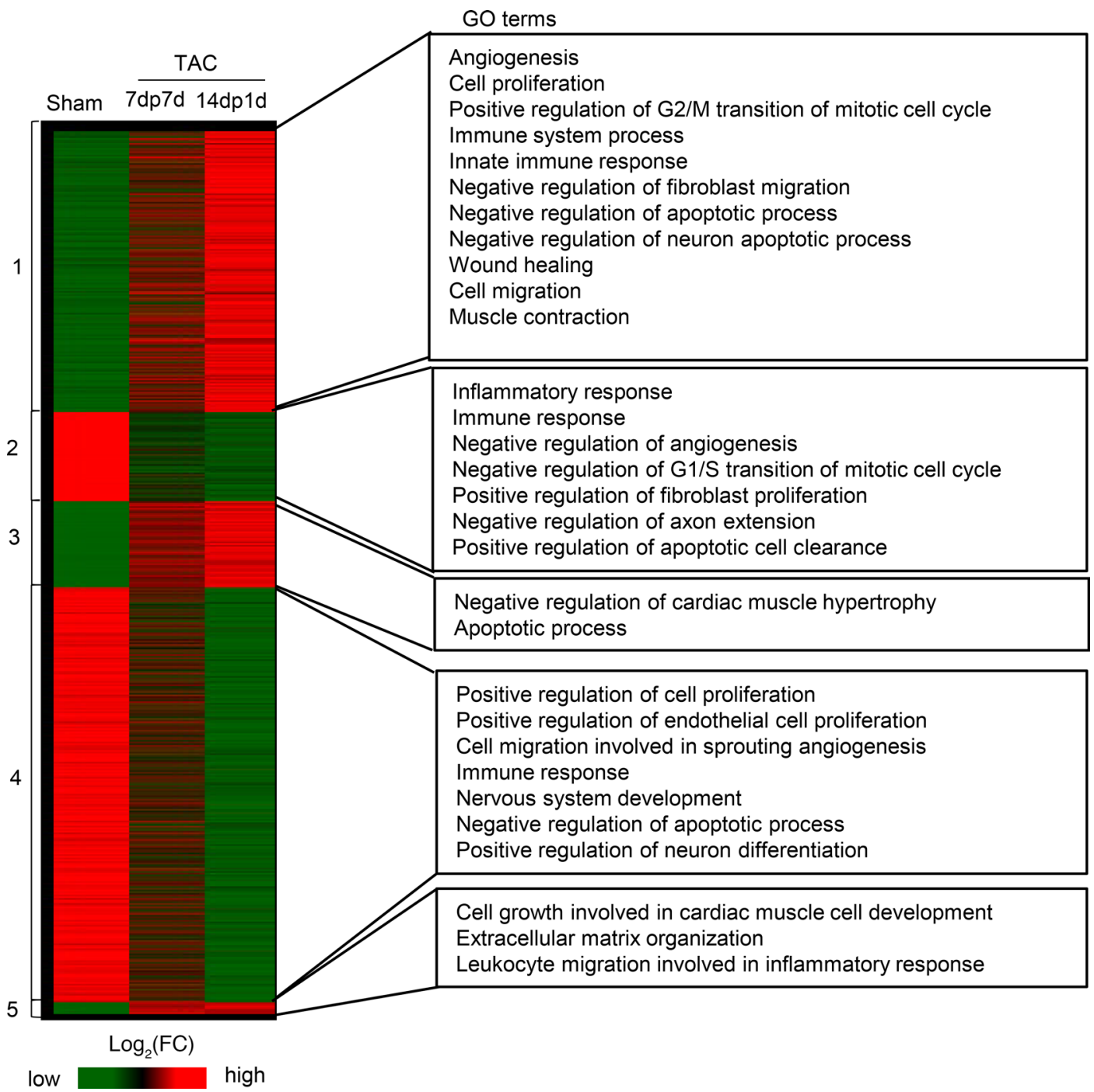

Figure 8. One-day-old mice, but not 7-day-old mice, upregulate a proregenerative gene expression pattern in the heart during nTAC. The heatmap representative of the top 5 clusters of differentially coregulated genes among sham, 7dp7dTAC, and 14dp1dTAC mice is shown. $n=3$ mice were analyzed by RNA sequencing per condition.

binding regions of GATA4 in the promoter region, we picked one of the regulated genes, Il6, as an example. As illustrated in Figure 11B, we identified high GATA4 binding peaks (highlighted in yellow) indicating the binding site of GATA4 in ChIP-seq on E12.5 with much smaller binding peaks in adult sham mice, although an increase in response to adult TAC was observed. The last 2 lines in Figure 11B show Il6 in our RNA-seq results, indicating increased expression in 14dp1dTAC versus 7dp7dTAC. To pinpoint GATA4 DNA binding regions in relation to the transcriptional start site (TSS) of the 696 upregulated genes, reanalysis of the cardiac GATA4 ChIP-seq from GSE52123 showed that GATA4 preferentially binds around the TSS in these genes. In addition, GATA4 DNA binding was most prominent during embryonic development (E12.5), when compared with adulthood, but it was, in part, reactivated during TAC at the adult stage (Figure 11C). Thus, this analysis suggests that upregulation of 696 genes in the regenerative (14dp1dTAC) compared with the nonregenerative phase (7dp7dTAC) during nTAC could be, in part, attributable to the high expression of GATA4 at this age.

Cardiomyocyte-specific ablation of GATA4 abrogates all features of the regenerative response to $n T A C$ immediately after birth. Since most of the upregulated genes in the regenerative phase after nTAC were GATA4 target genes, we performed nTAC in 1-day-old cardiomyocyte specific GATA4 KO (CM-G4-KO: $\operatorname{Tg}[\beta-M H C-C r e] ; G a t a 4^{1 / / 1}$ mice) and control mice (Gata4 $4^{\mathrm{A} / \mathrm{l}}$ littermates) to address the functional role of GATA4 for the regenerative response to neonatal pressure overload (Figure 12A). Previous analyses of 
14dp1dTAC vs 7dp7dTAC

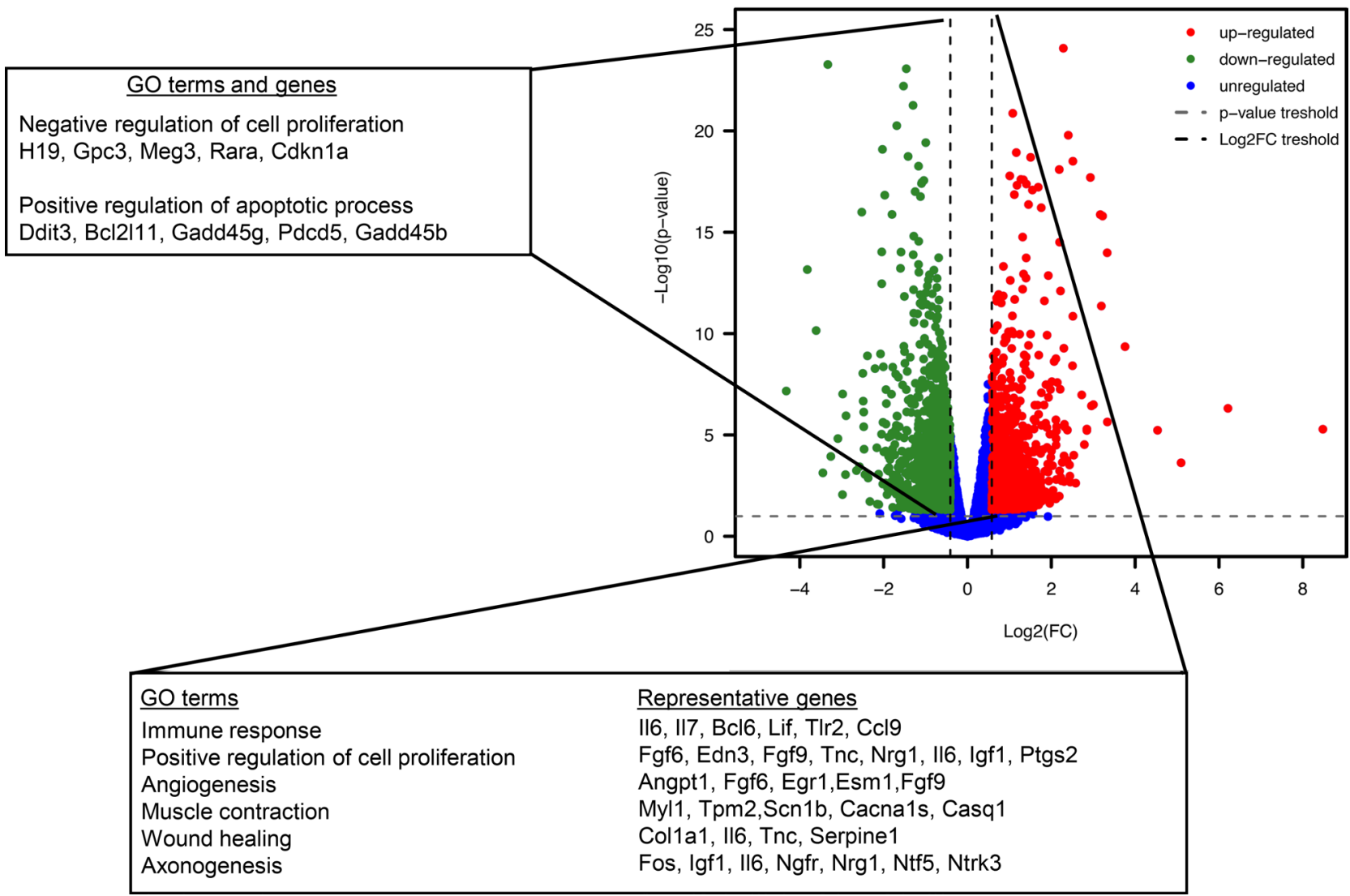

Figure 9. A proregenerative gene-expression pattern is observed in regenerative nTAC. Volcano plot showing differentially expressed genes in hearts of 14dp1dTAC versus 7dp7dTAC mice. Representative gene ontology (CO, biological processes) classes and example genes are shown. GO classes and genes upregulated in 14dp1dTAC are shown at the bottom, and downregulated GO classes/genes are shown on the left.

the CM-G4-KO mouse strain employed in our study had revealed that cardiomyocyte GATA4 deletion occurs shortly before birth around E18 and that these mice exert normal heart function and histology at least until the age of 8 weeks (17). During the first 2 weeks after nTAC on P1, however, Kaplan-Meier survival analysis showed that mortality in the CM-G4-KO mice was significantly increased compared with control mice (Figure 12B). The HW/BW ratios increased in both experimental groups after nTAC compared with their corresponding sham-operated mice (Figure 12C). Echocardiography revealed a reduced EF in CM-G4-KO mice compared with control mice after nTAC (Figure 12D). In addition, CM-G4-KO mice exhibited a significant increase in nTAC-induced fibrosis versus control mice (Figure 12E). Studying the regenerative process by immunofluorescence staining of heart sections revealed a reduced capillary density, as well as diminished cardiomyocyte mitosis (pH3) and cytokinesis (Aurora B midbody staining) after nTAC in CM-G4-KO mice (Figure 13, A-E). Interestingly, an increase in cardiomyocyte cross-sectional area was observed in CM-G4-KO mice after nTAC compared with control nTAC mice (Figure $13 \mathrm{~F})$. These data suggest that 1-day-old mice cannot trigger cardiomyocyte proliferation in the absence of GATA4; instead, they induce cardiomyocyte hypertrophy. This is associated with reduced cardiac function and significant interstitial cardiac fibrosis. Analysis of cardiac innervation showed a reduced abundance of nerves in CM-G4-KO hearts after nTAC (Figure 13G).

\section{Discussion}

According to current thinking, the nature of the overload stimulus mainly determines the reaction of the heart during the emergence of hypertrophy: physiological stimuli (such as pregnancy or regular strenuous exercise) lead to physiological hypertrophy, while disease stimuli (such as pressure overload or myocardial infarction) entail pathological hypertrophy $(1,2,25)$. In contrast with pathological hypertrophy, physiological 
A

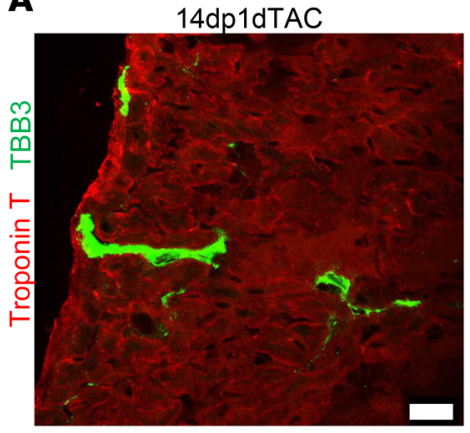

B

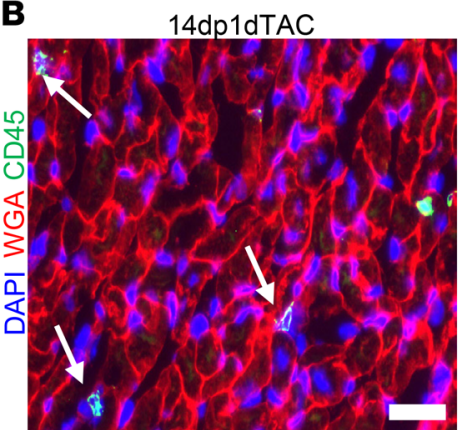

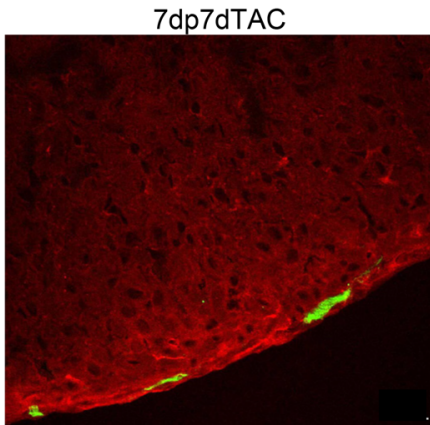

$7 d p 7 d T A C$

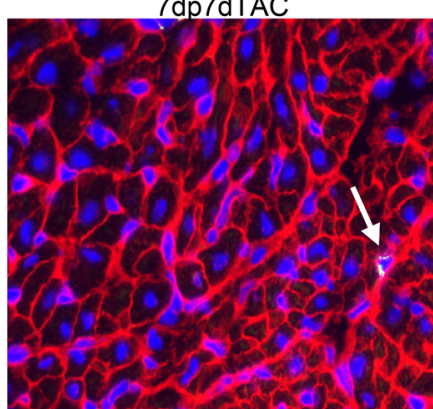

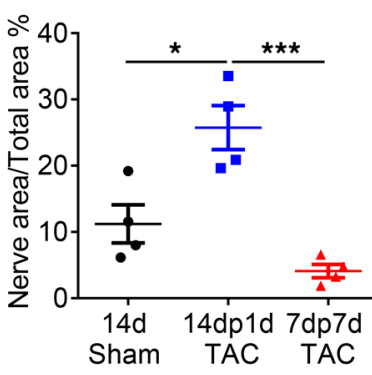

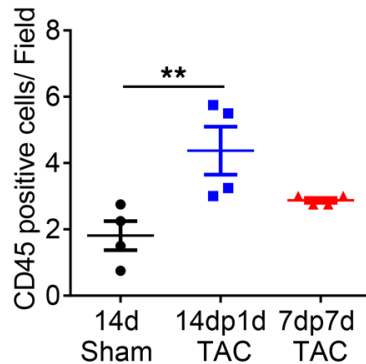

Figure 10. Increased innervation and inflammation after nTAC in 1-day-old, but not 7-day-old, mice. (A) Immunofluorescence staining of 14dp1dTAC and 7dp7dTAC mouse heart sections for the neurite marker class III $\beta$-tubulin (TBB3), and the quantification of neurite-covered area per total cardiac area in each group; $n=4$ per group. Scale bar: $20 \mu \mathrm{m}$. ${ }^{*} P<0.05,{ }^{* *} P<0.001$ (1-way ANOVA/Sidak's multiple comparison's test). (B) Immunofluorescence staining of 14dp1dTAC and 7dp7dTAC mouse heart sections for the leukocyte marker CD45, and quantification of leukocytes on the right; $n=4$ per group. Scale bar: $20 \mu \mathrm{m}$. ${ }^{* *} P<0.005$ (Kruskal Wallis test/Dunn's multiple comparison's test). Arrows indicate CD45 positive cells.

hypertrophy is not accompanied by cardiac dysfunction and myocardial fibrosis, but by enhanced myocardial angiogenesis and cardiomyocyte proliferation (26). Here, we show that a pathological stimulus, when applied shortly after birth, can also induce a purely adaptive response that is very similar to physiological hypertrophy. This indicates that the reaction of the heart to external stimuli is profoundly dependent on the intrinsic molecular wiring of the heart, which strongly changes during postnatal growth and maturation. Indeed, a recent study by the group of Enzo R. Porrello indicated that gene expression in cardiac cells changes more between injury induction on P1 versus induction in the nonregenerative adult stage than between uninjured and injured hearts at the same age (27). Accordingly, gene expression profiling in our study revealed a much stronger induction of genes promoting cellular proliferation, angiogenesis, inflammation, muscle contraction, and innervation when nTAC was conducted at day 1 (14dp1d TAC) versus when nTAC was induced in the nonregenerative stage at day 7 (7dp7TAC) after birth.

Our data suggest that cardiomyocyte proliferation, in contrast with cellular hypertrophy, promotes adaptation during pressure overload. Rapamycin administration — which inhibits cell proliferation, for instance in isolated cardiomyocytes, during cardiac development and in cancer models $(22,28-30)$ induced mortality and myocardial fibrosis as a sign of insufficient regeneration after nTAC. Quite similarly, inhibition of cardiomyocyte proliferation by other means, for instance through transgenic overexpression of Meis1 or miR-195, leads to fibrosis and cardiac dysfunction after myocardial infarction in the neonatal regenerative time frame $(31,32)$. The deleterious role of rapamycin stands in contrast with its beneficial effects during pressure overload in adult mice when no cardiomyocyte proliferation occurs and when it ameliorates cardiac dysfunction by blocking pathological cardiomyocyte hypertrophy (33). The adaptive role of cardiomyocyte proliferation during pressure overload is supported by the finding that myocardial overexpression of cyclin D2 promotes cardiomyocyte proliferation during TAC in adult mice, thereby upholding cardiac function and counteracting fibrosis, while at the same time limiting cardiac hypertrophy (34). We also observed in our study that the occurrence of cardiomyocyte proliferation prevented maladaptive cardiomyocyte hypertrophy, while in turn, inhibition of cardiomyocyte proliferation 
A

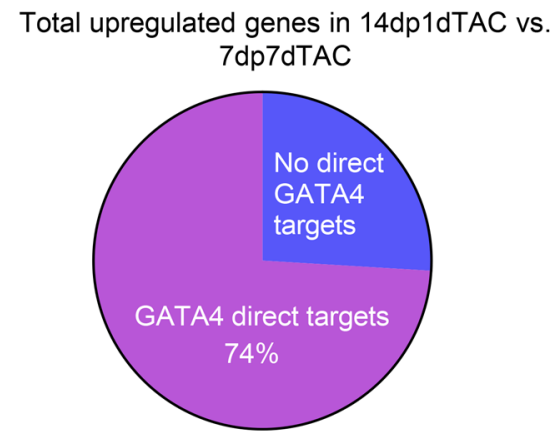

C

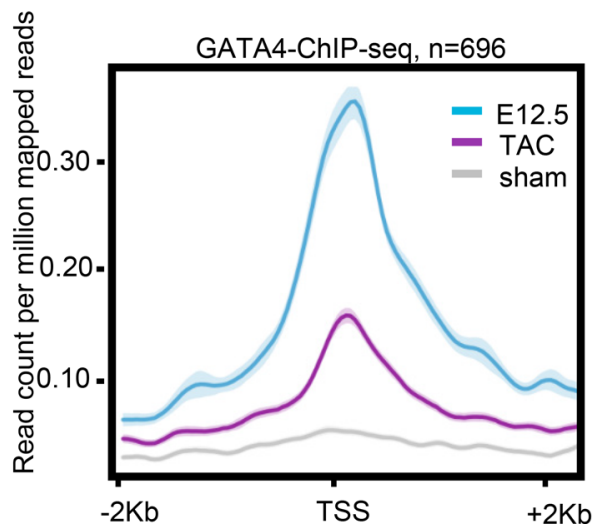

B

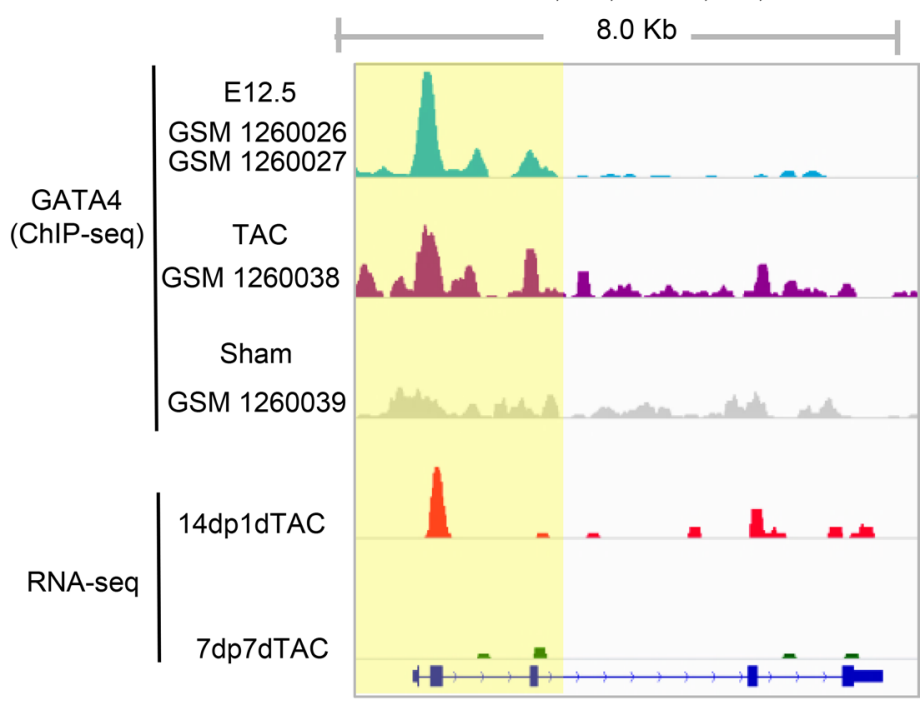

$1 / 6$

Figure 11. The majority of upregulated genes in the regenerative phase (14dp1dTAC) compared with the nonregenerative phase (7dp7dTAC) are directly bound by GATA4. (A) Pie chart illustrating that $74 \%$ of the upregulated genes in 14dp1dTAC compared with 7dp7dTAC are directly bound by GATA4 according to the published GATA4 ChIP sequencing data (GSE52123). (B) Visualization of GATA4 ChIP sequencing of E12.5, adult sham, and TAC, as well as RNA sequencing data tracks from 14dp1dTAC and 7dp7dTAC mice for II6. (C) Average profile of CATA4 ChIP-seq-derived GATA4 binding with regard to the transcriptional start site (TSS) from GSE52123 on the suggested targets of GATA4 (696 upregulated genes) in 14dp1dTAC compared with 7dp7dTAC mice.

(due to the deletion of GATA4 or due to PTK787 treatment) led to cardiomyocyte hypertrophy and cardiac dysfunction. Because angiogenesis was also inhibited by rapamycin, we could not differentiate between the influences of cardiomyocyte proliferation versus vessel growth for the regenerative response to pressure overload. To address this, we employed PTK787 as angiogenesis inhibitor (35). Indeed, PTK787 strongly reduced myocardial angiogenesis during nTAC. However, while we verified that PTK787 did not directly impair cellular proliferative activity in cardiomyocyte monoculture, it reduced cardiomyocyte proliferation and blunted regeneration after nTAC in the regenerative stage in vivo. This led us to conclude that in vivo angiogenesis is needed to support cardiomyocyte proliferation, that both processes are closely intertwined during the regenerative response to pressure overload, and that they might act in concert to promote myocardial regeneration under these circumstances.

In addition to enhanced cardiomyocyte proliferation and angiogenesis, we observed increased myocardial leukocyte abundance and enhanced myocardial innervation in the regenerative versus nonregenerative nTAC model. Studies have shown an inevitable role of the immune system for the induction of cardiomyocyte proliferation and angiogenesis in 1-day-old mice and zebrafish following myocardial infarction and apex resection (36-41). Immunosuppression of 1-day-old mice, for example, abrogates the cardiac regenerative capacity after apex resection, due to the absence of IL-6 (41). Accordingly, we found that immune response genes such as $I l 6$ were downregulated in nonregenerative (7dp7dTAC) compared with the regenerative phase pressure overload (14dp1dTAC). While IL-6 is capable of directly inducing cardiomyocyte proliferation (40, 42, 43), other inflammatory mediators (such as $\mathrm{Cc1} 9$ and Tlr2) upregulated in regenerative nTAC might act indirectly 
A

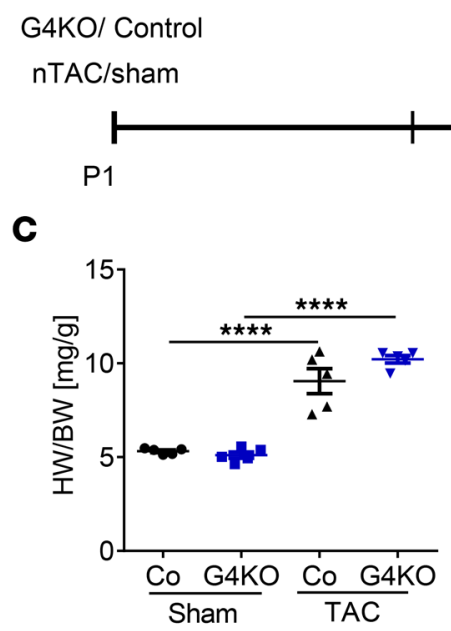

E

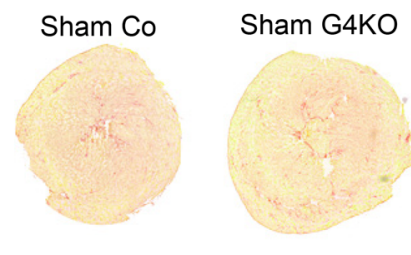

Echocardiography and sacrifice

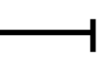

$\mathrm{P} 14$

D

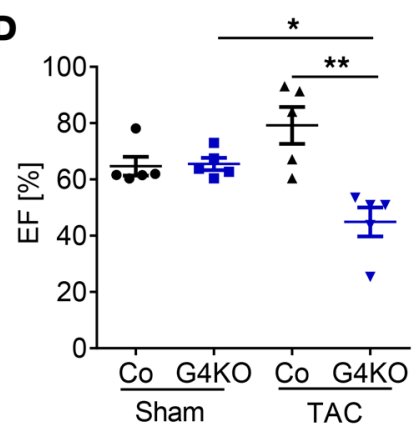

B

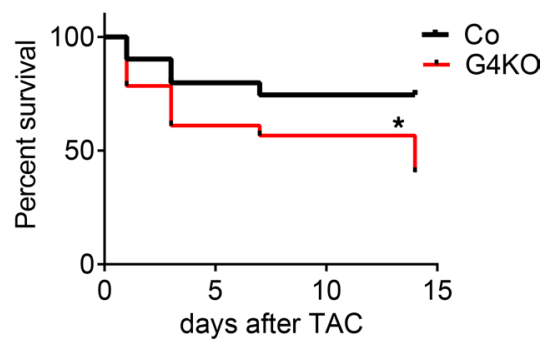

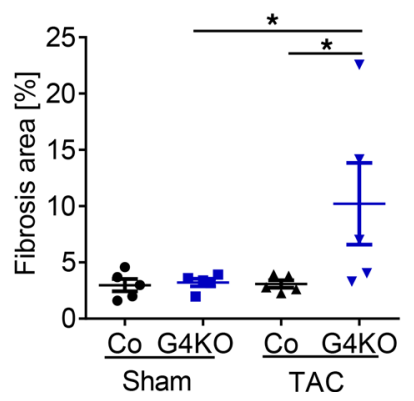

Figure 12. Pressure overload triggers cardiac failure in neonatal G4-KO mice. (A) A schematic representation of the experimental time schedule. (B) Kaplan-Meier survival curve of G4-KO and control (Co) mice after nTAC at P1; $n=15$, Co; $n=24$, G4-KO. ${ }^{*} P<0.05$ (Gehan-Breslow-Wilcoxon test). (C) HW/ BW ratio in G4-KO and Co mice after sham or TAC surgery; $n=5$ per group. ${ }^{* * *} P<0.0001$ (1-way ANOVA/Sidak's multiple comparison's test). (D) Ejection fraction (EF) measured by echocardiography in G4-KO and Co sham and TAC mice; $n=5$ per group. ${ }^{*} P<0.05,{ }^{* *} P<0.005$ (Kruskal Wallis test/Dunn's multiple comparison's test). (E) Sirius Red staining of heart sections from the indicated mice (scale bar: $500 \mu \mathrm{m})$ and quantification of the fibrotic area, $n=5$ per group. ${ }^{*} P<0.05$ (1-way ANOVA/Sidak's multiple comparison's test). Co, Control; G4-KO, cardiomyocyte specific GATA4 KO mice (CM-C4-KO).

by recruiting monocytes into the myocardium, which in turn promote angiogenesis $(37,44,45)$. Pressure overload at the adult stage in mice is also associated with increased abundance of inflammatory cells (mainly macrophages), although controversy still exists as to whether they play an adaptive or maladaptive role during TAC (46-48). With regard to nerves, myocardial tissue is innervated to regulate heart function. Cardiac pressure overload in adult mice reduced myocardial nerve density, and a similar pattern is observed in human failing myocardium (49-51). In contrast, in our regenerative pressure overload model, an increased myocardial axonogenesis was detected, which might promote cardiac contractility (e.g., through the release of norepinephrine) and - on the other hand - promote cardiomyocyte proliferation by secreting factors such as neuregulin-1 $(52,53)$. Indeed, a recent study showed that cardiac regeneration following myocardial infarction in 1-day-old mice is accompanied by dense hyperinnervation in the injured area (54). Pharmacological inhibition of sympathetic or parasympathetic nerve activity, as well as mechanical cholinergic denervation, inhibits heart regeneration of neonatal mice and zebrafish, owing to the inability of cardiomyocytes to proliferate; this suggests a high impact of nerves on the myocardial regenerative capacity of 1-day-old mice $(36,54)$. Therefore, although we do not demonstrate a causal link between enhanced myocardial nerve growth and the regenerative response during nTAC, we suggest that both processes are closely related, functionally.

In order to be able to induce regeneration in patients suffering from pressure overload-induced cardiomyopathy in the future, one needs to identify crucial upstream regulators that promote this regenerative response. We have previously shown that GATA4 is highly abundant in the myocardium of 1-day-old mice and that it is essential for cardiac regeneration after myocardial infarction at this age, but its expression is dramatically reduced by P7 (15). Crossing our RNA-seq results from the current study with published myocardial GATA4 ChIP-seq data revealed that the majority (74\%) of the upregulated genes in the regenerative compared with the nonregenerative phase after nTAC are bound by GATA4 in proximity to their TSS 
A

A DAPI WGA IB4

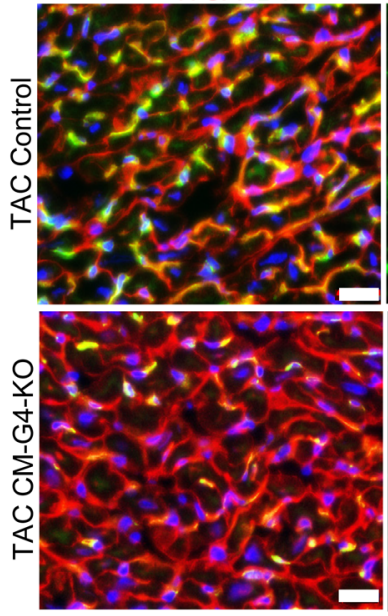

B

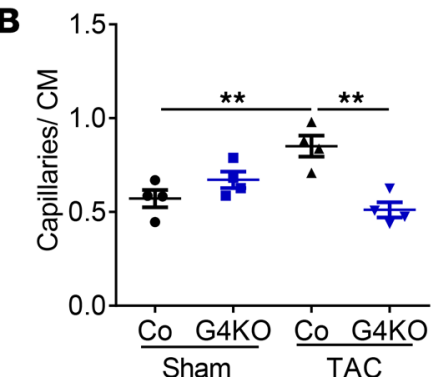

$\mathbf{E}$

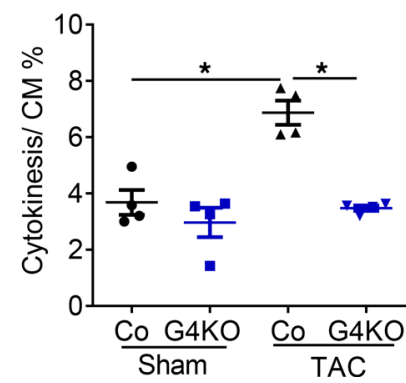

C
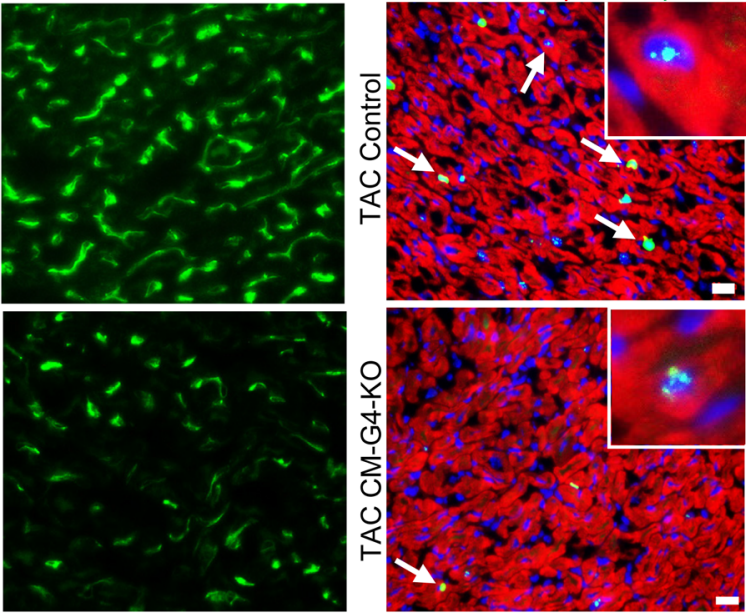

D

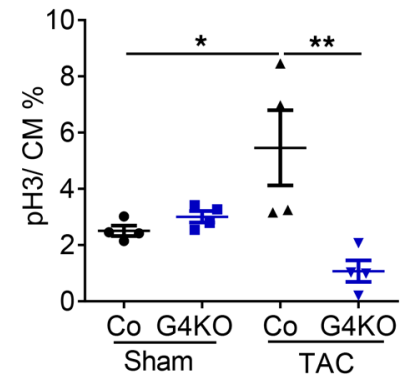

$F$

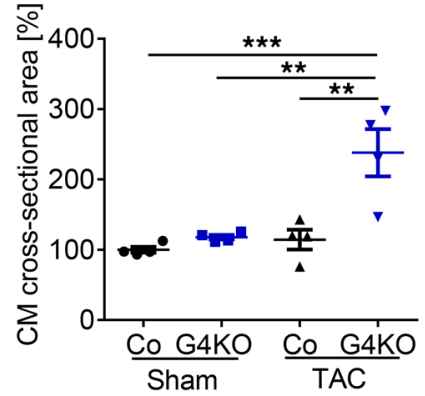

G

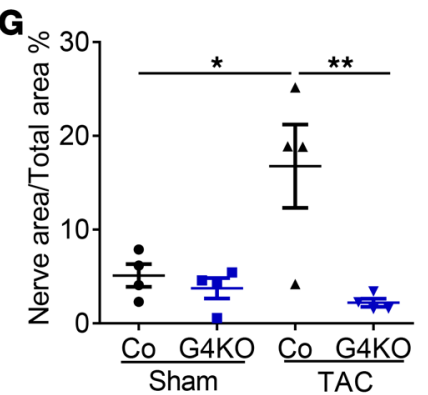

Figure 13. Angiogenesis and cardiomyocyte proliferation are not induced following nTAC in G4-KO mice. (A and B) Immunofluorescence stained heart sections for isolectin B4 (IB4), WGA, and DAPI (A) and quantification of the capillaries/cardiomyocyte ratio (B); $n=4$ per group. Scale bar: $20 \mu \mathrm{m}$. ${ }^{* *} P$ $<0.005$ (1-way ANOVA/Sidak's multiple comparison's test). (C) Immunofluorescence staining of heart sections for pH3, Troponin T, and DAPI. Arrows indicate $\mathrm{pH} 3$-positive cardiomyocytes. Inserts represent higher magnification of pH3 positive cardiomyocytes. (D) Quantification of pH3-positive cardiomyocytes; $n=4$ per group. Scale bar: $20 \mu \mathrm{m}$. ${ }^{*} P<0.05$, ${ }^{* *} P<0.005$ (1-way ANOVA/Sidak's multiple comparison's test). (E and F) Quantification of cytokinesis per cardiomyocyte; ${ }^{*} P<0.05$ (Kruskal Wallis test/Dunn's multiple comparison's test) and of the cardiomyocyte cross-sectional area in the indicated mice; $n=4$ per group. ${ }^{* *} P<0.005$, ${ }^{* *} P<0.001$ (1-way ANOVA/Sidak's multiple comparison's test). (G) Quantification of nerve-covered area in G4-KO and control hearts after TAC and sham operation; $n=4$ per group. ${ }^{*} P<0.05,{ }^{* *} P<0.005$ (1-way ANOVA/Sidak's multiple comparison's test). Co, control; G4-KO, cardiomyocyte specific GATA4 KO mice (CM-G4-KO).

and are therefore likely direct targets of GATA4 (24). Remarkably, the GATA4 target genes represented the majority of all GO functional classes observed in the genes upregulated during regenerative pressure overload. These data suggest GATA4 as master regulator of the regenerative nTAC response. Indeed, cardiomyocyte-specific ablation of GATA4 converted the myocardial response to nTAC at P1 into a nonregenerative response, as it is usually only seen after P7. While the adaptive, as well as proangiogenic, role of cardiomyocyte GATA4 has been previously recognized in adult TAC (17-19), its function to promote cardiomyocyte proliferation (during nTAC) and myocardial axonogenesis had not been described before, to our knowledge. Reactivation of the regenerative program in adult cardiomyocytes is mainly hampered by inaccessible chromatin in the region of regenerative genes (27). Given the potency of GATA4 as a transcriptional regulator - including its function to act as pioneering factor (55), which is able to bind DNA even in genomic regions of closed chromatin to induce profound changes in cellular fate - it might be 
reasonable to speculate that delivery of GATA4 could be a therapeutic strategy for pressure overload in the future. Further understanding of the mechanisms behind the ability of 1-day-old mice to compensate for pressure overload will be needed and may uncover valuable therapeutic strategies to treat or prevent maladaptive cardiac hypertrophy in patients.

\section{Methods}

Experimental animals. ICR-CD1 mice were obtained from Charles River Laboratories. Male and female neonatal mice were equally used throughout the study in all experimental groups. The mice with cardiomyocyte-specific GATA4 deletion (CM-G4-KO: Tg[B-MHC-Cre]; GATA4 ${ }^{\mathrm{f} / \mathrm{I}}$ ) were previously described and were maintained on a mixed SV129/CD1 background (17). Gata4 $4^{\mathrm{f} / \mathrm{fl}}$ littermates were used as control mice for the CM-G4-KO mice. The animals had free access to water and a standard diet and were maintained on a 12-hour light and dark cycle at room temperature $\left(22^{\circ} \mathrm{C} \pm 2^{\circ} \mathrm{C}\right.$ ) (maintenance diet, ssniff, V1534-300; breeding diet, ssniff, V1124-300).

nTAC surgery and treatments. After induction of anesthesia with $4 \%-5 \%$ isoflurane, mice were fixed with tape on an ice pad to conduct the surgery. Partial thoracotomy at the second rib was performed under a surgical microscope. The thymus was pulled aside for identification of the aortic arch. A 10-0 suture (Ethilon, EH7467G) was inserted under the transverse aorta between the innominate and LCA. A 33G needle (Nanofil, NF33BL 33GA, blunt needle) was placed in parallel to the transverse aorta. Three knots were tied around the transverse aorta and the needle. The needle was then gently removed, and the skin and muscles were closed using a 8-0 absorbable suture (Vicryl, V542G). The mice were warmed under a heating lamp and placed back into the mother's cage. For sham surgery, mice were treated similarly but without the induction of aortic constriction. The mother received water $(500 \mathrm{ml})$ including NovaMin 200 (50 drops) from 1 day before surgery until 3 days after surgery. The perioperative mortality after nTAC surgery was around $20 \%$.

Rapamycin (MilliporeSigma, RO395, $2 \mathrm{mg} / \mathrm{kg}$ per day) or solvent (0.2\% sodium carboxymethylcellulose and $0.25 \%$ polysorbate- 80 in water) was injected i.p. every day after surgery for 7 days. PTK787 (Abmol, M1882, $25 \mathrm{mg} / \mathrm{kg}$ ) or DMSO were i.p. injected from days 7-10 after surgery.

Echocardiography and Doppler flow velocity measurement. For echocardiography, mice were anesthetized with $0.5 \%-1.0 \%$ isoflurane and placed on a heating pad to maintain body temperature, as described (15). Noninvasive, echocardiographic parameters were measured with a linear 30-MHz transducer (Vevo 3100, Visualsonics). LVEDA and the average diastolic wall thickness were recorded or calculated from the parasternal short axis view. The EF was calculated from the long parasternal axis view.

Following echocardiography, the flow velocity signals of the RCA and the LCA were measured by placing the Doppler-probe (20 MHz probe of INDUS instruments; Version 1.7) on the right or left side of the cervical midline, respectively (56).

Histological analysis. The hearts were fixed in 4\% paraformaldehyde and were embedded in paraffin. Sirius Red staining was performed on 7- $\mu \mathrm{m}$ sections, as previously described (15). Subsequently, the fraction of the fibrotic area (stained in red) from the total myocardial area was determined using Adobe Photoshop Imaging Software.

Immunofluorescence staining and analyses. Hearts were frozen in OCT medium and cut into 7- $\mu \mathrm{m}$ sections. Sections were fixed in 4\% paraformaldehyde for 20 minutes, washed 3 times with PBS, and permeabilized with $0.3 \%$ Triton in PBS for 30 minutes. The slides were blocked in 3\% BSA for 30 minutes and then incubated with a specific primary antibody (pH3, MilliporeSigma, 06-570, diluted 1:200 in blocking solution); Troponin T (Abcam, 8295, 1:200); Aurora B (MilliporeSigma, A5102, 1:200); class III $\beta$-tubulin (TBB3, Abcam, 18207, 1:200); CD45 (Santa Cruz Biotechnology Inc., 25590, 1:50); Ki67 (Abcam, 15580, 1:100); ad Nkx2.5 (Santa Cruz Biotechnology Inc., H-114, 1:100) overnight at $4^{\circ} \mathrm{C}$. On the following day, the sections were washed 3 times with PBS and incubated with a corresponding secondary antibody, conjugated to Alexa 555 or Alexa 488 (1:200, Invitrogen) for 1 hour. Staining was also performed with WGA-Texas Red (Invitrogen, w21405, 1:100) and/or IB4 (Vector Laboratories, FL-1201, 1:50) for 1 hour. The sections were washed and mounted with mounting medium containing DAPI (Vector Laboratories, H-1500). Immunofluorescence analysis of tissue sections was performed using a confocal microscope (Leica DM IRB with a TCS SP2 AOBS scan head). After omission of the primary antibodies, no specific immunolabeling was observed.

Quantification of immunofluorescence images was performed at 400× magnification in 4-8 randomly selected high-power fields per animal. Confocal microscopy images after class III $\beta$-tubulin immunostaining were quantified to evaluate innervation density as ratio of nerve-covered myocardial area and total 
myocardial area (by ImageJ software; NIH). Confocal microscopy images were used to quantify $\mathrm{pH} 3$ and Aurora B-positive staining. Cytokinesis was analyzed by quantifying the ratio of midbody Aurora B staining in cardiomyocytes versus the total number of cardiomyocytes. For quantification of myocardial capillary density, the number of capillaries per cardiomyocyte was calculated.

Cell culture. Neonatal rat cardiomyocyte were isolated from 1- to 3-day-old neonatal rats, as previously described (15). The cells were treated with either $500 \mathrm{ng} / \mathrm{ml}$ rapamycin or solvent $(0.2 \%$ sodium carboxymethyl cellulose and $0.25 \%$ polysorbate- 80 in water; MilliporeSigma) or $500 \mathrm{ng} / \mathrm{ml}$ PTK or DMSO (NEB) for 24 hours in the presence of $15 \%$ FBS. On the following day, the cells were fixed with $100 \%$ ethanol for 5 minutes (Roth), permeabilized with $0.3 \%$ Tween 20 (Roth) for 5 minutes, washed with PBS (Thermo Fisher Scientific), blocked with 3\% BSA (MilliporeSigma) for 20 minutes, and incubated with a specific primary antibody (pH3, MilliporeSigma, 06-570, 1:200; Troponin T, Abcam, 8295, 1:200; Aurora B, MilliporeSigma, A5102, 1:200) overnight. On the next day, the cells were washed and incubated with a secondary antibody for 1 hour. After washing with PBS, the cells were covered with mounting medium and a cover slip.

$R N A$-seq and bioinformatics analysis. The left ventricle was isolated from 14dp1dTAC, 7dp7dTAC, and sham-operated mice. Total RNA was extracted by RNeasy mini kit according to the manufacture's protocol (Qiagen; 217004). Quality and integrity of total RNA was verified using the Agilent Technologies 2100 Bioanalyzer (Agilent Technologies). The RNA-seq library was generated from 200 ng total RNA using Dynabeads mRNA DIRECT Micro Purification Kit (Thermo Fisher Scientific) for mRNA purification followed by ScriptSeq v2 RNA-Seq Library Preparation Kit (Epicentre) according to manufacturer's protocols. The libraries were sequenced on Illumina HiSeq2500 using TruSeq SBS Kit v3-HS (50 cycles, single-ended run) with an average of $3 \times 10^{7}$ reads per RNA sample. Before alignment to the reference genome, each sequence in the raw FASTQ files was trimmed on base call quality and sequencing adapter contamination using the Trim Galore! wrapper tool. Reads shorter than 20 bp were removed from FASTQ files. Trimmed reads were aligned to the reference genome using the open source short read aligner STAR (https://code. google.com/p/rna-star). Feature counts were determined using the R package Rsubread. Only genes showing counts greater than 5 at least 2 times across all samples were considered for further analysis (data cleansing). Gene annotation was done by the $\mathrm{R}$ package bioMaRt. Before starting the statistical analysis steps, expression data were $\log _{2}$ transformed and normalized (according to the 50th percentile). Differential gene expression was calculated by the $\mathrm{R}$ package edgeR. A gene was considered to be differentially regulated when $P<0.05$ and $0.58 \leq \log _{2} \mathrm{FC} \leq-0.41$. Analysis of differentially regulated functional gene classes was performed by using DAVID 6.8 online tools. RNA-seq data are available at GEO (GSE121308).

The GATA4 ChIP-seq data were taken from GSE52123 (24). Trimmed reads were mapped to the mouse reference genome mm10 (UCSC assembly) using the default settings of bowtie2 (PMID 22388286). GATA4 enriched areas (peaks) were determined by using model-based analysis for ChIPseq (MACS14.1, p1e-3, PMID 18798982). Peaks were annotated by using annotePeaks.p1 from Homer (PMID 20513432). Bigwig files were normalized to the genome with the help of bam Coverage (-bs 20 --smoothLength 40) from deepTools2 (PMID 24799436). Venn diagram between GATA4 ChIP-seq and RNA-seq were crossed by gene name using The KNIME 2.9.1 platform. An aggregation plot was generated by ngs.plot (https://github.com/shenlab-sinai/ngsplot; commit ID 3fa5311, branch default) (57). K-means from $\mathrm{R}$ was used to cluster differentially expressed genes from sham, 7d7TAC, and 14d1dTAC. The heatmap was generated using Morpheus web-based tools (https://software.broadinstitute.org/morpheus). A row normalization of $\log _{2} \mathrm{FC}$ from each gene was performed. A k-means of 5 was used because it was the number of cluster in the samples that showed the highest difference in gene expression after nTAC.

Statistics. Statistical analysis was performed using Prism 7 (GraphPad Software). Data are shown as mean \pm SEM. Sample size was chosen as a result of previous experience regarding data variability in similar models. No statistical method was used to predetermine sample size. All experiments were carried out in at least 3 biological replicates. The experiments were not randomized. The investigators were blinded for mouse genotype and treatment during surgeries, echocardiography, organ weight determination, and all histological and immunofluorescence quantifications. Premature death was a preestablished criterion for exclusion from an ongoing mouse experiment. A Shapiro-Wilk normality test was performed for all experimental groups before statistical comparisons were conducted. Only when all groups passed the normality test for a given parameter, they were compared by 1-way repeated-measures 
ANOVA, followed by the Sidak's multiple comparisons test or by an unpaired, 2-sided Student's $t$ test when comparing 2 experimental groups. When one of the groups did not pass the normality test, all groups were compared using the Kruskal Wallis test, followed by the Dunn's multiple comparisons test or by the Mann-Whitney $U$ test. Mouse mortality was analyzed by the Gehan-Breslow-Wilcoxon test. Differences were considered significant when $P<0.05$.

Study approval. All procedures involving the use and care of animals were performed according to the Guide for the Care and Use of Laboratory Animals published by the National Research Council (NIH Publication No. 85-23, revised 1996) and the German animal protection code. All animal procedures described in this study were approved by the local state authorities (the Lower Saxony State Office for Consumer Protection and Food Safety and the Regional Council Baden-Wuertemberg, Germany, file numbers: 33.12-42502-04-17/2588 and G-178/18).

\section{Author contributions}

MMM and JH designed the study, planned all experiments, and analyzed the data. MMM, AA, AI, YX, JC, AS, AG, ME, and RG performed experiments and analyzed data. ME, FAT, GD, and JB gave advice for the project, analyzed data, and critically revised the manuscript. MMM and JH wrote the manuscript. $\mathrm{JH}$ supervised the study and all experimentation. All authors read and approved the manuscript.

\section{Acknowledgments}

This study was supported by the Deutsche Forschungsgemeinschaft through the Cluster of Excellence REBIRTH (EXC62/3), the Heisenberg Program (HE3658/6-1and HE3658/6-2), and a research grant HE 3658/11-1.

Address correspondence to: Joerg Heineke, MD, Department of Cardiovascular Research (HKF), European Center for Angioscience, Medical Faculty Mannheim, Heidelberg University, Ludolf-Krehl Street 7-11, 68167 Mannheim, Germany. Phone: 49.621.383.71855; Email: joerg.heineke@medma.uni-heidelberg.de.

1. Heineke J, Molkentin JD. Regulation of cardiac hypertrophy by intracellular signalling pathways. Nat Rev Mol Cell Biol. 2006;7(8):589-600.

2. Hill JA, Olson EN. Cardiac plasticity. NEngl J Med. 2008;358(13):1370-1380.

3. Rockman HA, et al. Segregation of atrial-specific and inducible expression of an atrial natriuretic factor transgene in an in vivo murine model of cardiac hypertrophy. Proc Natl Acad Sci USA. 1991;88(18):8277-8281.

4. Hein S, et al. Progression from compensated hypertrophy to failure in the pressure-overloaded human heart: structural deterioration and compensatory mechanisms. Circulation. 2003;107(7):984-991.

5. Une D, et al. Clinical Impact of Changes in Left Ventricular Function After Aortic Valve Replacement: Analysis From 3112 Patients. Circulation. 2015;132(8):741-747.

6. Douglas PS, et al. Hemodynamic outcomes of transcatheter aortic valve replacement and medical management in severe, inoperable aortic stenosis: a longitudinal echocardiographic study of cohort B of the PARTNER trial. J Am Soc Echocardiogr 2015;28(2):210-7.e1.

7. Porrello ER, et al. Transient regenerative potential of the neonatal mouse heart. Science. 2011;331(6020):1078-1080

8. Porrello ER, Olson EN. A neonatal blueprint for cardiac regeneration. Stem Cell Res. 2014;13(3 Pt B):556-570

9. Uygur A, Lee RT. Mechanisms of Cardiac Regeneration. Dev Cell. 2016;36(4):362-374.

10. Haubner BJ, et al. Complete cardiac regeneration in a mouse model of myocardial infarction. Aging (Albany NY). 2012;4(12):966-977.

11. Yuan X, Braun T. Multimodal Regulation of Cardiac Myocyte Proliferation. Circ Res. 2017;121(3):293-309.

12. Ye L, et al. Early Regenerative Capacity in the Porcine Heart. Circulation. 2018;138(24):2798-2808.

13. Zhu W, et al. Regenerative Potential of Neonatal Porcine Hearts. Circulation. 2018;138(24):2809-2816.

14. Haubner BJ, et al. Functional Recovery of a Human Neonatal Heart After Severe Myocardial Infarction. Circ Res. 2016;118(2):216-221.

15. Malek Mohammadi M, et al. The transcription factor GATA4 promotes myocardial regeneration in neonatal mice. EMBO Mol Med. 2017;9(2):265-279.

16. Molkentin JD. The zinc finger-containing transcription factors GATA-4, -5 , and -6 . Ubiquitously expressed regulators of tissue-specific gene expression. J Biol Chem. 2000;275(50):38949-38952.

17. Oka T, et al. Cardiac-specific deletion of Gata4 reveals its requirement for hypertrophy, compensation, and myocyte viability. Circ Res. 2006;98(6):837-845.

18. Heineke J, et al. Cardiomyocyte GATA4 functions as a stress-responsive regulator of angiogenesis in the murine heart. J Clin Invest. 2007;117(11):3198-3210.

19. Bisping E, et al. Gata4 is required for maintenance of postnatal cardiac function and protection from pressure overload-induced heart failure. Proc Natl Acad Sci USA. 2006;103(39):14471-14476.

20. Yu W, et al. GATA4 regulates Fgf16 to promote heart repair after injury. Development. 2016;143(6):936-949.

21. Gupta V, Gemberling M, Karra R, Rosenfeld GE, Evans T, Poss KD. An injury-responsive gata4 program shapes the zebrafish 
cardiac ventricle. Curr Biol. 2013;23(13):1221-1227.

22. Burton PB, Yacoub MH, Barton PJ. Rapamycin (sirolimus) inhibits heart cell growth in vitro. Pediatr Cardiol. 1998;19(6):468470.

23. Layman DL. Growth inhibitory effects of dimethyl sulfoxide and dimethyl sulfone on vascular smooth muscle and endothelial cells in vitro. In Vitro Cell Dev Biol. 1987;23(6):422-428.

24. He A, et al. Dynamic GATA4 enhancers shape the chromatin landscape central to heart development and disease. Nat Commun 2014;5:4907.

25. Grund A, Heineke J. Exercise makes the difference: deconstructing physiological hypertrophy in swine. J Mol Cell Cardiol. 2015;79:89-91.

26. Boström $\mathrm{P}$, et al. C/EBP $\beta$ controls exercise-induced cardiac growth and protects against pathological cardiac remodeling. Cell. 2010;143(7):1072-1083.

27. Quaife-Ryan GA, et al. Multicellular Transcriptional Analysis of Mammalian Heart Regeneration. Circulation. 2017;136(12):1123-1139.

28. Lang SA, et al. Mammalian target of rapamycin is activated in human gastric cancer and serves as a target for therapy in an experimental model. Int J Cancer. 2007;120(8):1803-1810.

29. Zhu Y, et al. Mechanistic target of rapamycin (Mtor) is essential for murine embryonic heart development and growth. PLoS ONE. 2013;8(1):e54221.

30. Stephan S, et al. Effect of rapamycin alone and in combination with antiangiogenesis therapy in an orthotopic model of human pancreatic cancer. Clin Cancer Res. 2004;10(20):6993-7000.

31. Mahmoud AI, et al. Meis1 regulates postnatal cardiomyocyte cell cycle arrest. Nature. 2013;497(7448):249-253.

32. Porrello ER, et al. Regulation of neonatal and adult mammalian heart regeneration by the miR-15 family. Proc Natl Acad Sci USA. 2013;110(1):187-192.

33. McMullen JR, et al. Inhibition of mTOR signaling with rapamycin regresses established cardiac hypertrophy induced by pressure overload. Circulation. 2004;109(24):3050-3055

34. Toischer $\mathrm{K}$, et al. Cardiomyocyte proliferation prevents failure in pressure overload but not volume overload. J Clin Invest. 2017;127(12):4285-4296.

35. Zangi L, et al. Modified mRNA directs the fate of heart progenitor cells and induces vascular regeneration after myocardial infarction. Nat Biotechnol. 2013;31(10):898-907.

36. Mahmoud AI, et al. Nerves Regulate Cardiomyocyte Proliferation and Heart Regeneration. Dev Cell. 2015;34(4):387-399.

37. Aurora AB, et al. Macrophages are required for neonatal heart regeneration. J Clin Invest. 2014;124(3):1382-1392.

38. Godwin JW, Pinto AR, Rosenthal NA. Macrophages are required for adult salamander limb regeneration. Proc Natl Acad Sci USA. 2013;110(23):9415-9420.

39. Lavine KJ, et al. Distinct macrophage lineages contribute to disparate patterns of cardiac recovery and remodeling in the neonatal and adult heart. Proc Natl Acad Sci USA. 2014;111(45):16029-16034.

40. Fang Y, Gupta V, Karra R, Holdway JE, Kikuchi K, Poss KD. Translational profiling of cardiomyocytes identifies an early Jak1/Stat3 injury response required for zebrafish heart regeneration. Proc Natl Acad Sci USA. 2013;110(33):13416-13421.

41. Han C, et al. Acute inflammation stimulates a regenerative response in the neonatal mouse heart. Cell Res. 2015;25(10):1137-1151.

42. Przybyt E, Krenning G, Brinker MG, Harmsen MC. Adipose stromal cells primed with hypoxia and inflammation enhance cardiomyocyte proliferation rate in vitro through STAT3 and Erk1/2. J Transl Med. 2013;11:39.

43. Zlatanova I, Pinto C, Silvestre JS. Immune Modulation of Cardiac Repair and Regeneration: The Art of Mending Broken Hearts. Front Cardiovasc Med. 2016;3:40.

44. Kortlever RM, et al. Myc Cooperates with Ras by Programming Inflammation and Immune Suppression. Cell. 2017;171(6):1301-1315.e14.

45. Xu Y, Zhou Y, Lin H, Hu H, Wang Y, Xu G. Toll-like receptor 2 in promoting angiogenesis after acute ischemic injury. Int J Mol Med. 2013;31(3):555-560.

46. Liao X, et al. Distinct roles of resident and nonresident macrophages in nonischemic cardiomyopathy. Proc Natl Acad Sci USA. 2018;115(20):E4661-E4669

47. Wang JW, et al. Leukocytic Toll-Like Receptor 2 Deficiency Preserves Cardiac Function And Reduces Fibrosis In Sustained Pressure Overload. Sci Rep. 2017;7(1):9193.

48. Patel B, Ismahil MA, Hamid T, Bansal SS, Prabhu SD. Mononuclear Phagocytes Are Dispensable for Cardiac Remodeling in Established Pressure-Overload Heart Failure. PLoS ONE. 2017;12(1):e0170781.

49. Lehmann LH, et al. Essential role of sympathetic endothelin A receptors for adverse cardiac remodeling. Proc Natl Acad Sci USA. 2014;111(37):13499-13504.

50. Mühlfeld C, Schipke J, Schmidt A, Post H, Pieske B, Sedej S. Hypoinnervation is an early event in experimental myocardial remodelling induced by pressure overload. J Anat. 2013;222(6):634-644

51. Kaye DM, Vaddadi G, Gruskin SL, Du XJ, Esler MD. Reduced myocardial nerve growth factor expression in human and experimental heart failure. Circ Res. 2000;86(7):E80-E84

52. D'Uva G, et al. ERBB2 triggers mammalian heart regeneration by promoting cardiomyocyte dedifferentiation and proliferation Nat Cell Biol. 2015;17(5):627-638.

53. Polizzotti BD, et al. Neuregulin stimulation of cardiomyocyte regeneration in mice and human myocardium reveals a therapeutic window. Sci Transl Med. 2015;7(281):281ra45.

54. White IA, Gordon J, Balkan W, Hare JM. Sympathetic Reinnervation Is Required for Mammalian Cardiac Regeneration. Circ Res. 2015;117(12):990-994.

55. Mayran A, Drouin J. Pioneer transcription factors shape the epigenetic landscape. J Biol Chem. 2018;293(36):13795-13804

56. Hartley CJ, Reddy AK, Madala S, Entman ML, Michael LH, Taffet GE. Doppler velocity measurements from large and small arteries of mice. Am J Physiol Heart Circ Physiol. 2011;301(2):H269-H278.

57. Shen L, Shao N, Liu X, Nestler E. ngs.plot: Quick mining and visualization of next-generation sequencing data by integrating genomic databases. BMC Genomics. 2014;15:284. 
